\title{
Development of Gamma Ray Imaging Cameras
}

\section{Proaress Repont}

\author{
for \\ Second Year \\ prepared by \\ David K. Wehe and Glenn F. Knoll \\ Department of Nuclear Engineering \\ University of Michigan \\ for \\ DOE Grant DE-FG07-90ERI2932

\section{DISCLAIMER}

This report was prepared as an account of work sponsored by an agency of the United States Government. Neither the United States Government nor any agency thereof, nor any of their employees, makes any warranty, express or implied, or assumes any legal liability or responsibility for the accuracy, completeness, or usefulness of any information, apparatus, product, or process disclosed, or represents that its use would not infringe privately owned rights. Reference herein to any specific commercial product, process, or service by trade name, trademark, manufacturer, or otherwise does not necessarily constitute or imply its endorsement, recommendation, or favoring by the United States Government or any agency thereof. The views and opinions of authors expressed herein do not necessarily state or reflect those of the United States Government or any agency thereof. 


\section{Progress Report}

\section{Table of Contents}

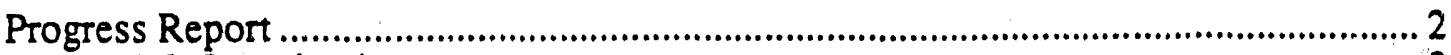

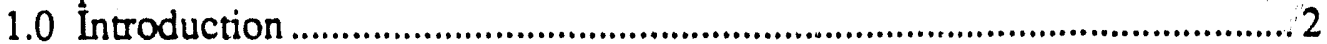

2.0 Mechanically-Collimated Cameras ................................................................... 2

2.1 Single-aperture raster scanning camera design and testing............... 2

2.1.1 Introduction ..................................................................... 2

2.1.2 Camera Design ................................................................. 3

2.1.3 Detector ...................................................................... 3

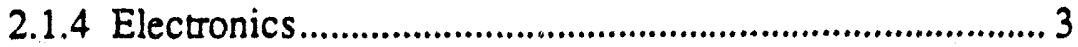

2.1.5 Image Display ............................................................ 4

2.2 Imaging results ........................................................................... 4

2.2.1 Realistic Environment Images ........................................ 4

2.3 Improvements in Detectors ............................................................ 5

2.3.1 Energy Resolution Measurements Using CsI with

PIN Photodiode ...........................................................................5

2.3.2 BaF2 with Wavelength Shifting ..................................... 8

3.0 Electronically-Collimated Cameras .............................................................. 16

3.1 Theoretical design .................................................................... 16

3.2 Reducing Azimuthal Ambiguity for Double Compton

Scattering through Polarization Information............................................. 24

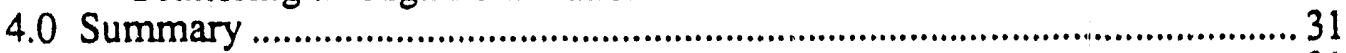

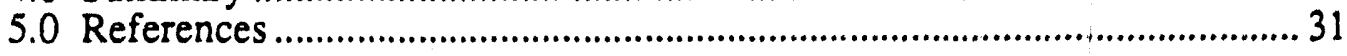




\section{Development of Gamma Ray Imaging Cameras}

\subsection{Introduction}

\section{Progress Repert}

In January 1990, the Department of Energy initiated this project with the objective to develop the technology for general purpose, portable gamma ray imaging cameras useful to the nuclear industry. The ultimate goal of this R\&D initiative is to develop the analog to the color television camera where the camera would respond to gamma rays instead of visible photons. The rwo-dimensional real-time image which would be displayed would indicate the geometric location of the radiation relative to the camera's orientation, while the brightness and "color" would indicate the intensity and energy of the radiation (and hence identify the emitting isotope). As we noted in our original proposal, there is a strong motivation for developing such a device for applications within the nuclear industry, for both high- and low-level waste repositories, for environmental restoration problems, and for space and fusion applications. At present, there are no general purpose radiation cameras capable of producing spectral images for such practical applications.

At the time of this writing, work on this project has been underway for almost 18 months. Substantial progress has been made in the project's two primary areas: mechanically-collimated (MCC) and electronically-collimated camera (ECC) designs. In section 2, we present developments covering the mechanically-collimated design, and section 3 discusses the efforts on the electronically-collimated camera. A summary of these accomplishments is contained in section 4 . The renewal proposal addresses the continuing R\&D efforts for the third year effort.

\subsection{Mechanically-Collimated Cameras}

The mechanically collimated camera uses physical barriers to form the apertures necessary in the imaging process, and is the favored method in medical imaging. In this section, we describe the first prototype and present results of images obtained from this singleaperture, raster scanning camera.

\subsection{Single-aperture raster scanning cankera design and testing}

\section{1 .1 Introduction}

In this section, we focus on the develophy of our portable radion imaging camera for imaging intense gamma ray fields. Thr 1 kortable imager is intended to be 
moved into a high radiation area, acquire the image, and send the data back to the operator. From a survey of potential applications and common isotopes, it was decided that the camera should be designed to cover the energy range of 0.1 to $3 \mathrm{MeV}$ and exposure rates up to $2 \mathrm{R} / \mathrm{hr}$. Applying principles from astrophysics and medical imaging cameras, along with physical constraint of mobility, we built and have now refined a single aperture raster scanning camera that meets these requirements. In the following sections, we review the camera principles, and present new images. During the past year, we have acquired images using the prototype camera, designed the second prototype camera, performed experiments on next generation detectors, and improved the functionality of the camera by replacing the SCA with an MCA and inline spectral analysis software.

\section{1 .2 Camera Design}

The camera consists of a shielded gamma ray detector mounted on a pan and tilt table. The purpose of the table is to position the camera aperture precisely. Images are formed by recording the count rate of a given isotope at each orientation of the aperture. By repeatedly moving the aperture and acquiring data, the image is formed pixel by pixel. Since only a single aperture is used, images are obtained without deconvolution.

\subsubsection{Detector}

The gamma ray detector of choice continues to be a $1.27 \mathrm{~cm} \times 1.27 \mathrm{~cm}$ right circular cylinder of bismuth germanate (BGO) which is coupled to a R1166 photomultiplier tube. $B G O$ was chosen for its high $Z$ value, having an intrinsic peak efficiency that is more than twice that of $\mathrm{NaI}(\mathrm{Tl})$. For a given crystal size, this implies that more full energy depositions will occur in the BGO than $\mathrm{NaI}(\mathrm{Tl})$. However, the lower gamma ray scintillation efficiency of $\mathrm{BGO}$, only $20 \%$ of $\mathrm{NaI}(\mathrm{Tl})$, leads to a slightly worse energy resolution than $\mathrm{NaI}(\mathrm{TI})$. The desire for compactness outweighed the advantage of improved energy resolution for this application, and thus BGO has remained as the detector element.

\subsection{Electronics}

The pulse processing electronics associated with the camera were modified during the past year by replacing the SCA, which permitted only single photon energy imaging, with an MCA. The MCA was installer internal to the IBM AT, so that spectra were obtained and peaks processed during each pixel acquisition. The software automatically adjusts for pulse height drifting during the image acquisition. We discovered that performing peak searches before doing the background subtraction improved the 
resolution of the images significantly. The result of this effort is an integrated image acquisition package which uses state-of-the-art peak search routines. This work was presented in detail in reference (1).

\subsubsection{Image Display}

The image data were acquired on an IBM AT computer. Thece data files were transferred to A pollo and/or DEC 3100 workstations for processing and display. Since the camera has good spatial resolution, processing simply involved extracting the net count rate and forming a normalized image file. With the new spectral capabilities, the user can request isotope-specific images. Since most of the images shown below had known incident energy, color coding was used to visually display intensity. Unless otherwise noted, the color palette chosen for display is a black body radiator, i.e., white is the most intense and deep red being the least intense.

\subsection{Imaging results}

During our first year, our primary attention was spent in developing the prototype camera and obtaining laboratory images. During the past twelve months, efforts were expended obtaining images in realistic environments around the Ford Nuclear Reactor (FNR) at the University of Michigan.

\subsubsection{Realistic Environment Images}

The accompanying video tape shows a sequence of realistic radioactive environments and associated radiation images. The tape begins with a $360^{\circ}$ pan of the FNR equipment room. The viewer should note the constrained spaces which dictate the need for portability, whereas the intense fields require remote operation. The tape focuses on the deionizing and holdup tanks and then shows the radiation images of these tanks. Note that the images permit localization of the $\sim R / h r$ hot spots from a distance of a 2-3 meters away.

The second part of the tape shows images of sources taken in the hot caves. Radioactive steel plates were arranged as shown on the tape, and the resulting radiation images were acquired. Of particular interest is the last image -- two steel plates surrounding a $\sim \mathrm{mCi}{ }^{137} \mathrm{Cs}$ source. For this image, the green color was chosen to represent the ${ }^{127} \mathrm{Cs}$ radiation, while the red color represented ${ }^{60} \mathrm{Co}$ radiation coming from the steel plates. Note the very clear separation in both space and energy of these three sources. 


\subsection{Improvements in Detectors}

While BGO coupled with a conventional PMT offers reasonable energy resolution, newer scintillators and light conversion devices may offer improved performance. We have initiated a program to evaluate and drive the development of such devices. Two particularly interesting scintillators are $\mathrm{CsI}$ and $\mathrm{BaF}_{2}$ coupled to $\mathrm{Si}$-PIN photodiodes or PMTs.

\subsubsection{Energy Resolution Measurements Using CsI with PIN Photodiode}

Our last progress report showed initial results of the optimization of gamma-ray energy resolution as a function of temperature for CsI(Tl) coupled to a silicon PIN photodiode (PD) (cf. Figure 1). During the past year, we have pursued alternate strategies to further explore the decrease in dark current (i.e., noise) realized by cooling the photodiode. Our investigations have revealed that each contributing component of the energy resolution must be more thoroughly understood in order to explain and further improve on the overall energy resolution optimization.

Figure 2 shows the our best results by cooling solely the CsI(TI)/PD. The improvement over our initial result is primarily due to a new CsI(TI) crystal that is polished and wrapped to optimize light collection efficiency. However, this improvement in energy resolution is still not sufficient to justify non-room temperature (RT) operation. The reason that that the PD cooling has not led to significant improvements in energy resolution is because the $\mathrm{CsI}(\mathrm{Tl})$ light output decreases with temperature. As a result, we tested cooling the PD while leaving the $\mathrm{CsI}(\mathrm{Tl})$ at $\mathrm{RT}$, as well as cooling the preamplifier (preamp) with the CsI(TI) and PD.

For the first case, we installed a light pipe which penetrated an environmental chamber and cooled the PD while maintaining the CsI(Tl) at RT. The light pipe transported light from the $\mathrm{CsI}(\mathrm{Tl})$ to the $\mathrm{PD}$, but the observed light transmission was only $30 \%$. In addition, the thermal barrier was not sufficient to prevent some cooling of the CsI crystal. The net result was the disappointing degradation in energy resolution shown in Figure 3.

Figure 4 shows the results of cooling the preamp with the CsI(Tl)/PD. This method improves energy resolution by decreasing noise. By shortening the distance between the preamp and PD the stray capacitance is decreased. Also, cooling the preamp input FET decreases its noise current and increases the forward transconductance. All three of these effects decrease noise and thus improve energy resolution. Unfortunately the temperature range of most commercial preamps only extend down to $0^{\circ} \mathrm{C}$ and thus prohibit cooling significantly below this temperature. Nevertheless, this method has 


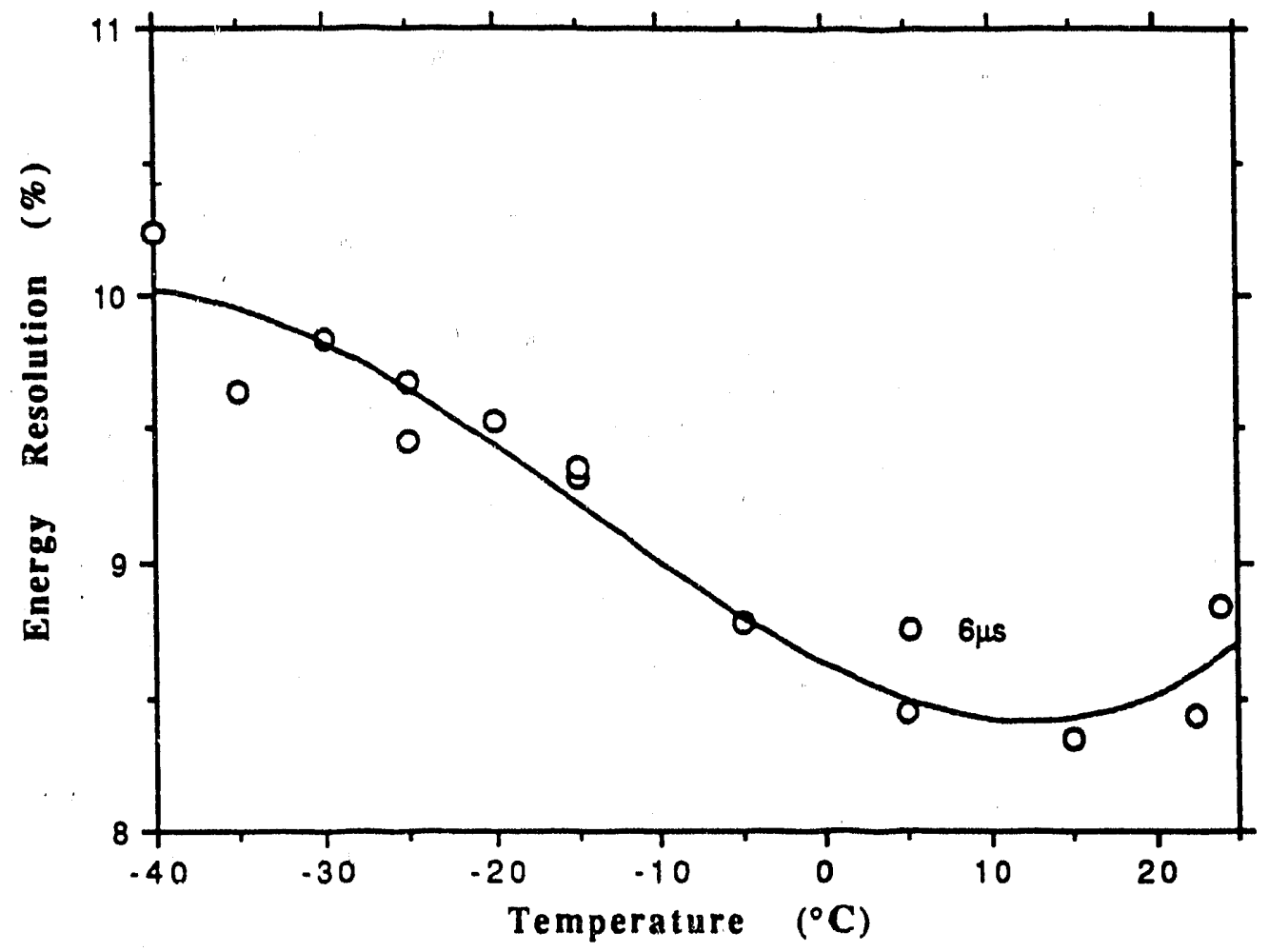

Figure 1: Preliminary Results - Gamma-ray Energy Resolution for CsI(TI)/PD Cooled

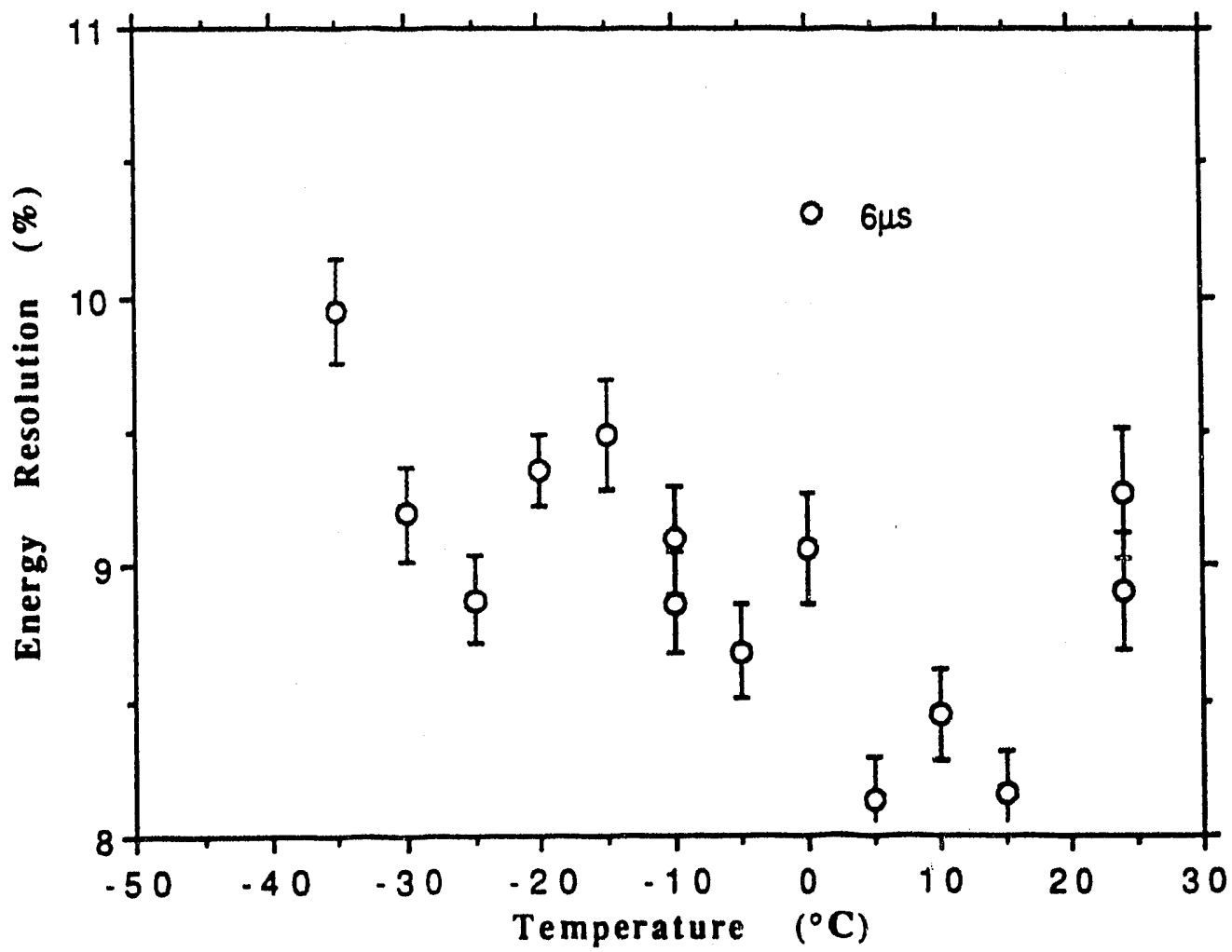

Figure 2: Best Results - Gamma-ray Energy Resolution for CsI(TI)/PD Cooled 


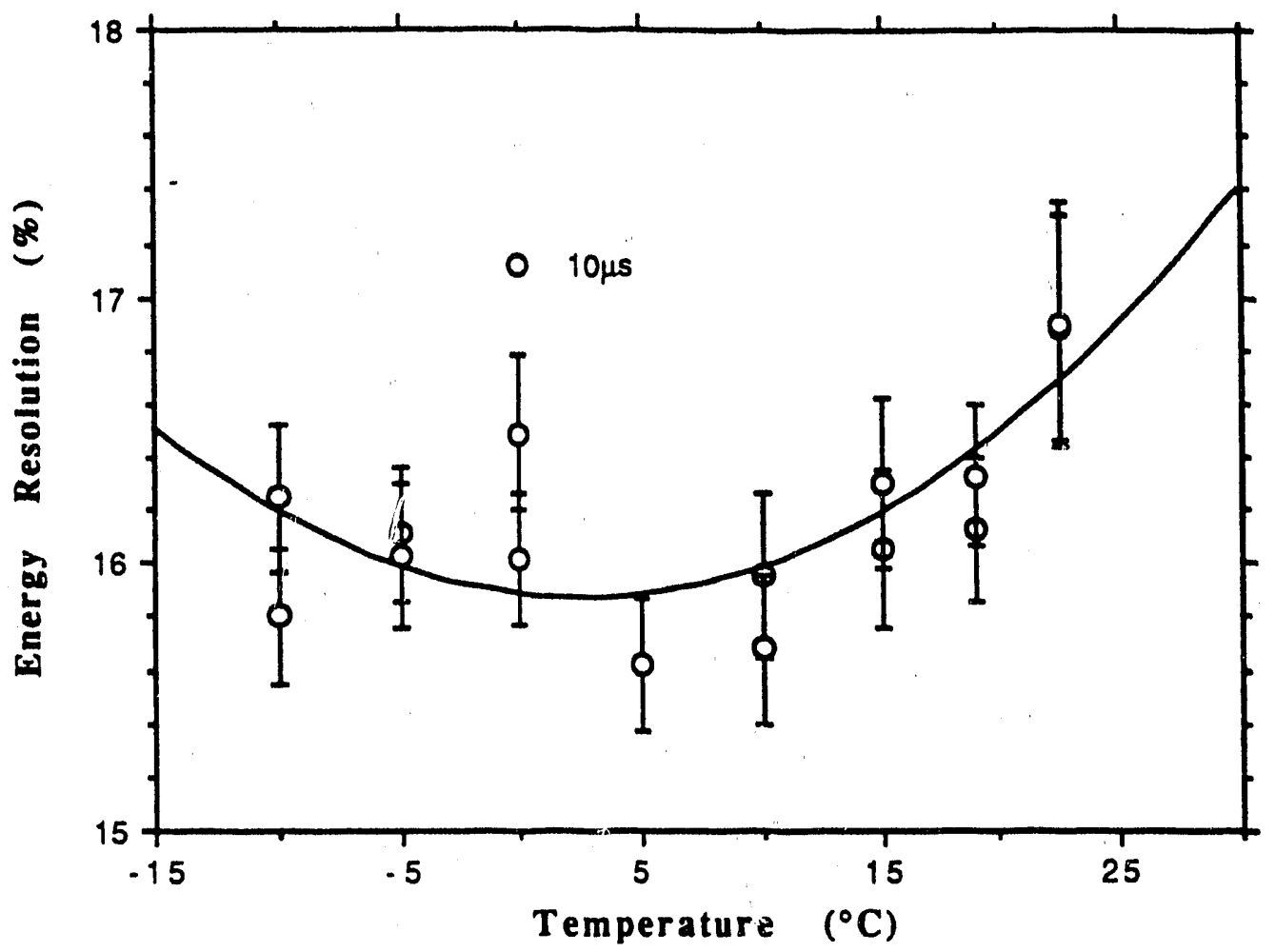

Figure 3: Best Results - Gamma-ray- Energy

Resolution for CsI(Tl) at RT, PD Cooled

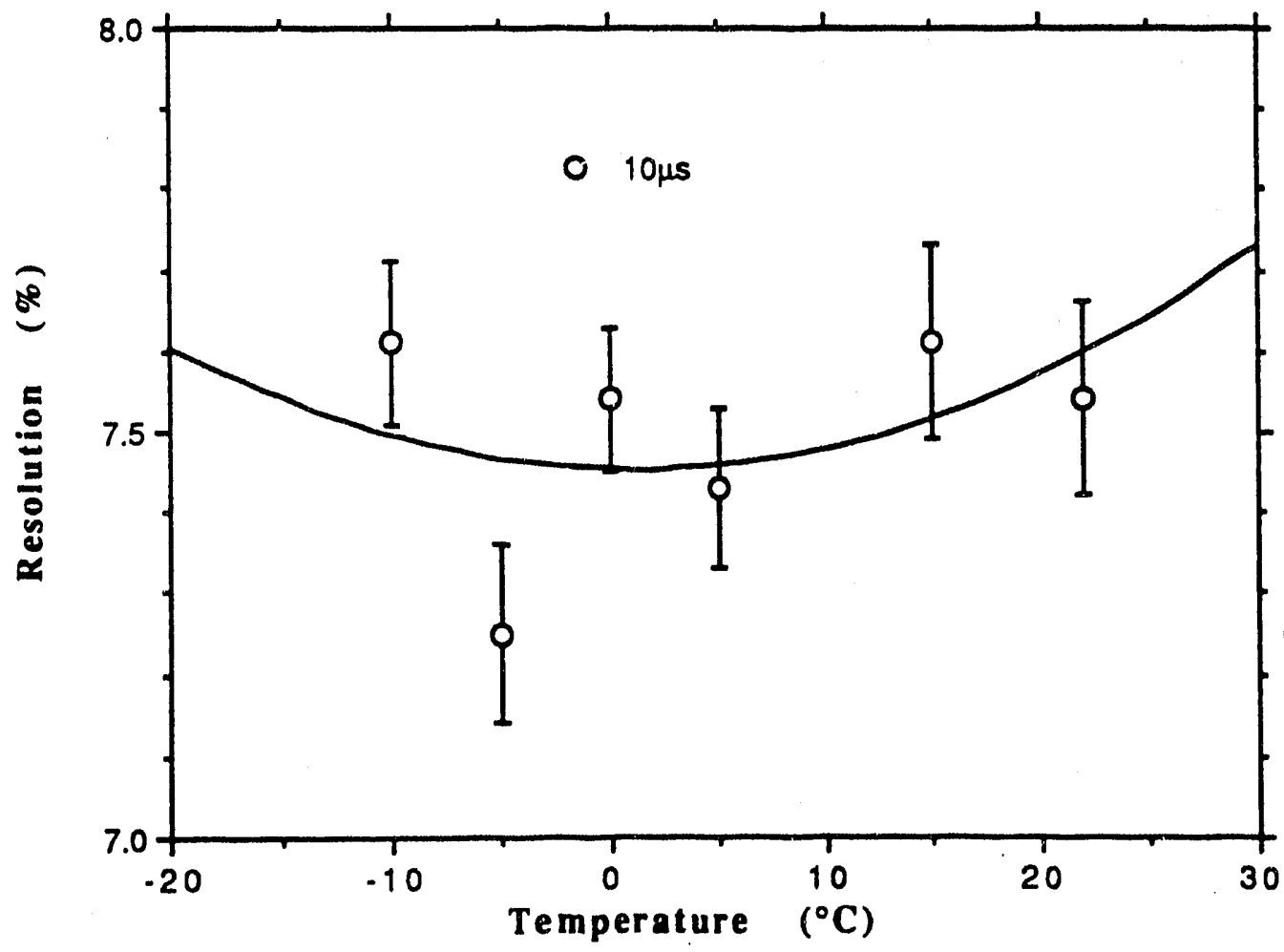

Figure 4: Best Results - Gamma-ray energy Resclution for CsI(TI)/PD/preamp Cooled 
proven to be the most reproducible and has yielded the best results.

While acquiring the energy resolution data, scintillation light yield data was also recorded. When our results were compared with published results small discrepancies were noticed in relative numbers. This prompted further investigation into scintiliation light output as a function of temperature. Consequently, absolute measurements have been made and are shown in Figure 5. Knowing the absolute scintillation light yield allows a calculation of the contribution to energy resolution due to statistical variations in the number of photons produced. These investigations have raised questions, which we are now addressing, regarding the use of some fundaniental properties of light collection efficiency and quantum efficiency in scintillation systems.

With the effects of the scintillation process in a gamma ray detector better understood, our attention has turned to the electronics (PD and preamp). Since the PD and preamp noise are the major contributors to energy resolution, it is important to know how to minimize these contributions while not degrading pulse height information. For instance, it has been determined that the best RT PD is not the best PD when cooled. This is because at RT the best PD has relatively low capacitance and dark current, but when cooled the dark current becomes negligible and the capacitance is the main source of noise. A PD with the lowest possible capacitance (even with a relatively high dark current at RT) will have superior noise properties at the cooler temperatures. Once the role of the electronics is better understood, a system with improved energy resolution should be possible.

\subsection{2 $\mathrm{BaF}_{2}$ with Wavelength Shifting}

With the first prototype gamma ray imaging camera operational and havirig the much needed capability to acquire spectral images, it was decided to begin the design of the second prototype. Several designs were considered: pinhole, multiple collimator, aciive background suppression shielding rather than the inactive lead shielding, and a design that utilized little to no shielding, only the shuttering mechanism. The final design is the most versatile, but puts the most stringent requirements on the detector element. In fact, all the proposed designs had one underlying necessity: a fast, dense, radiation hard detector. This would allow the camera to be used in higher radiation fields than is obtainable with the current camera. Noting that the radiaticn detector is the most critical element in the camera design, basic detector development has been stressed during the past twelve months.

Researchers in medical imaging and high energy physics have also been interested in developing a fast, dense, radiation hard detector. For the medical imaging groups, this 


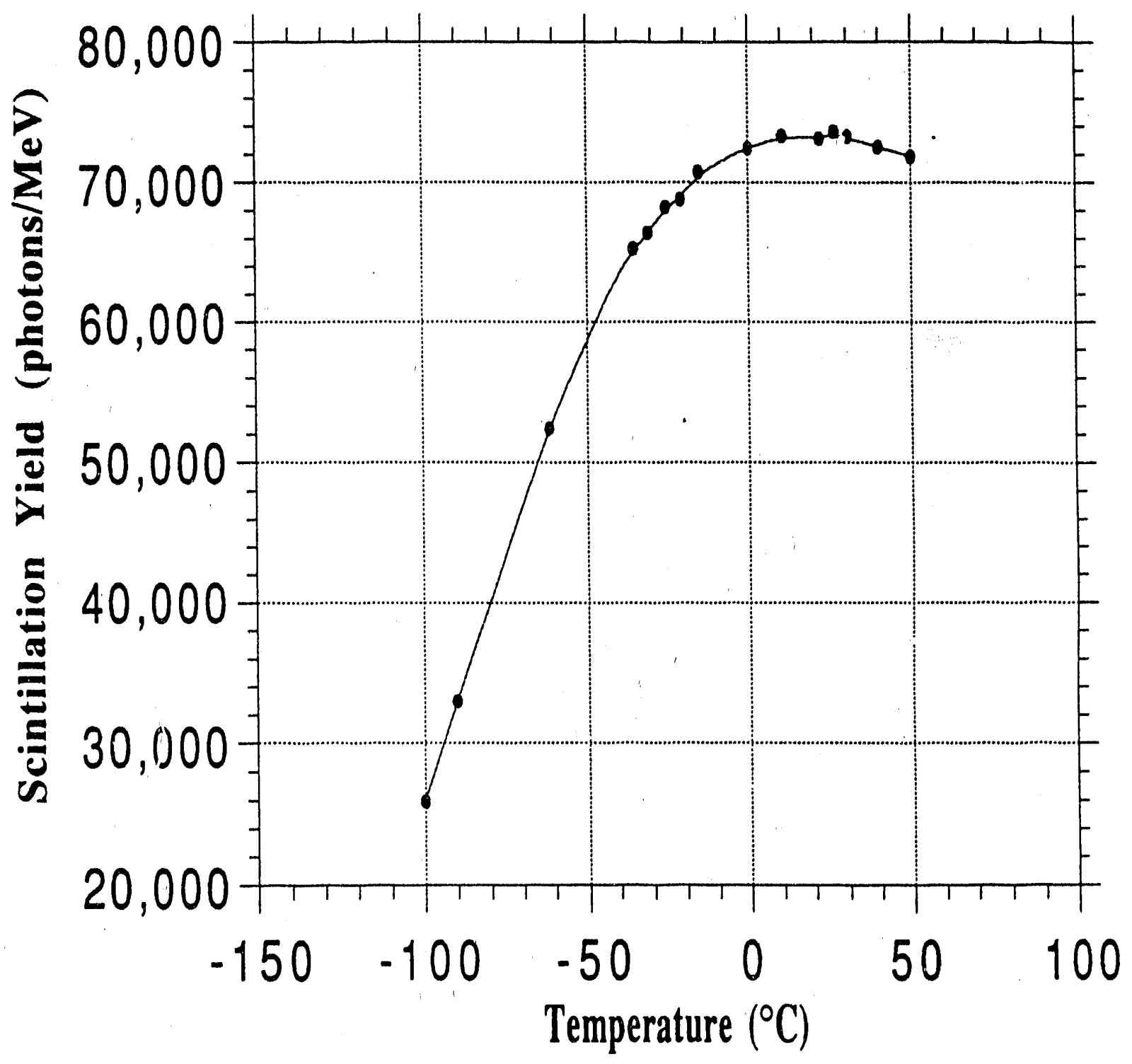

Figure 5: Temperature Dependent Absolute Scintillaiion Yield of CsI(TI) 
type of detector would allow them to extend the capabilities for Positron Emission Tomography (PET) to Time-of-Flight PET. This would permit enhanced spatial resolution to $1 \mathrm{~mm}$. High energy physics researchers are interested in such a detector for detection of charged particles at the rate of $10^{8} \mathrm{cps}$. The type of detector that is suitable for the atove applications could also be used as the detection element in the next generation gamma ray imaging camera. Over the last couple of years, researchers have discovered several fast, dense scintillators, but their luminosity (i.e., emission intensity) has been low. Compounds such as $\mathrm{PbSO}_{4}, \mathrm{PbCO}_{3}, \mathrm{CeF}_{3}, \mathrm{LaF}_{3}(\mathrm{Nd})$ were discovered and $\mathrm{BaF}_{2}$ has remained under active investigation to better utlize the fast component of its emission $\left(\mathrm{BaF}_{2}\right.$ is the fastest known inorganic scintillator). One of the key research groups that was involved with the discovery and characterization of $\mathrm{PbSO}_{4}, \mathrm{PbCO}_{3}$, and $\mathrm{CeF}_{3}$, along with various other inorganic compounds, is a group at Lawrence Berkeley Laboratory ( $\mathrm{LBL}$ ), headed by Stephen Derenzo. Last summer, one of our senior graduate students worked with the group from LBL as they investigated the scintillation properties of some 430 inorganic compounds. This research was conducted at the National Synchrotron Light Source at the Brookhaven National Laboratory. It was at this time that several potential scintillators were discovered, including one with comparable luminosity to $\mathrm{CsI}(\mathrm{Tl})$ but with a single decay constant of $10 \mathrm{~ns}$. This compound's ultimate viability has yet to be determined.

Despite these inten/se investigations, $\mathrm{BaF}_{2}$ remains the fastest inorganic scintillator. It is for this reason we have decided to concentrate our efforts on the the development of a scintillation detection system that utilizes $\mathrm{BaF}_{2} . \mathrm{BaF}_{2}$ has at least two emissions that peak at $220 \mathrm{~nm}$ and $310 \mathrm{~nm}$. The $220 \mathrm{~nm}$ emission has a decay time of 0.6 $\mathrm{ns}$ where as the $310 \mathrm{~nm}$ emission has a decay constant of $600 \mathrm{~ns}$. Most researchers have exploited the speed of $\mathrm{BaF}_{2}$ by using special fused silica endwindow photomultiplier tubes (PMT) which transmit this fast ultraviolet emission, while others have used regular borosilicate PMTs and wavelength shifters to detect the fast $220 \mathrm{~nm}$ emission.

Wavelength shifting is a teclinique in whicl. the scintillator emissions are absorbed by an organic dye. Within a few nanoseconds, the organic deexcites and emits a photon having a longer wavelength (less energy) which is more readily detected. Figure 6 illustrates the effect of using p-Terphenyl (PTP) as a wavelength shifter. The open diamond symbol illustrates the amount of light that is detected with just coupling compound between the scintillator and the borosilicate PMT. If a wavelength shifter is utilized, the number of detected photons ircreases, hence moving the peak to a higher channel number. The amount of increase is dependent on the solvent (due to dipole-dipole interactions between the solvent and the solute), the quantum efficiency of the wavelength shifter and the 
quantum efficiency of the PMT at the emission wavelength of the wavelength shifter. Toluene is known to have good energy transfer to PTP and hence has the highest light output of any of the solvent/solute combinations shown here. Figure 7(a) shows the scintillation decay curve of $\mathrm{BaF}_{2}$ as measured with a borosilicate endwindow PMT. From the slope of the curve, we can determine that the scintillation decay time is $600 \mathrm{~ns}$ for the slow component of $\mathrm{BaF}_{2}$. Figure 7(b) shows two components of light, a spike at $90 \mathrm{~ns}$ (from the wavelength shifted fast emission of $\mathrm{BaF}_{2}$ ), as well as the slow emission. Figure 8 shows how the energy spectrum is changed due to the introduction of PTP. The curve of open circles shows the coupling of $\mathrm{BaF}_{2}$ to the PMT with no wavelength shifter and the closed circles show the increase in the amount of light detected (shifting the spectrum to the right) by introducing the organic dye, PTP.

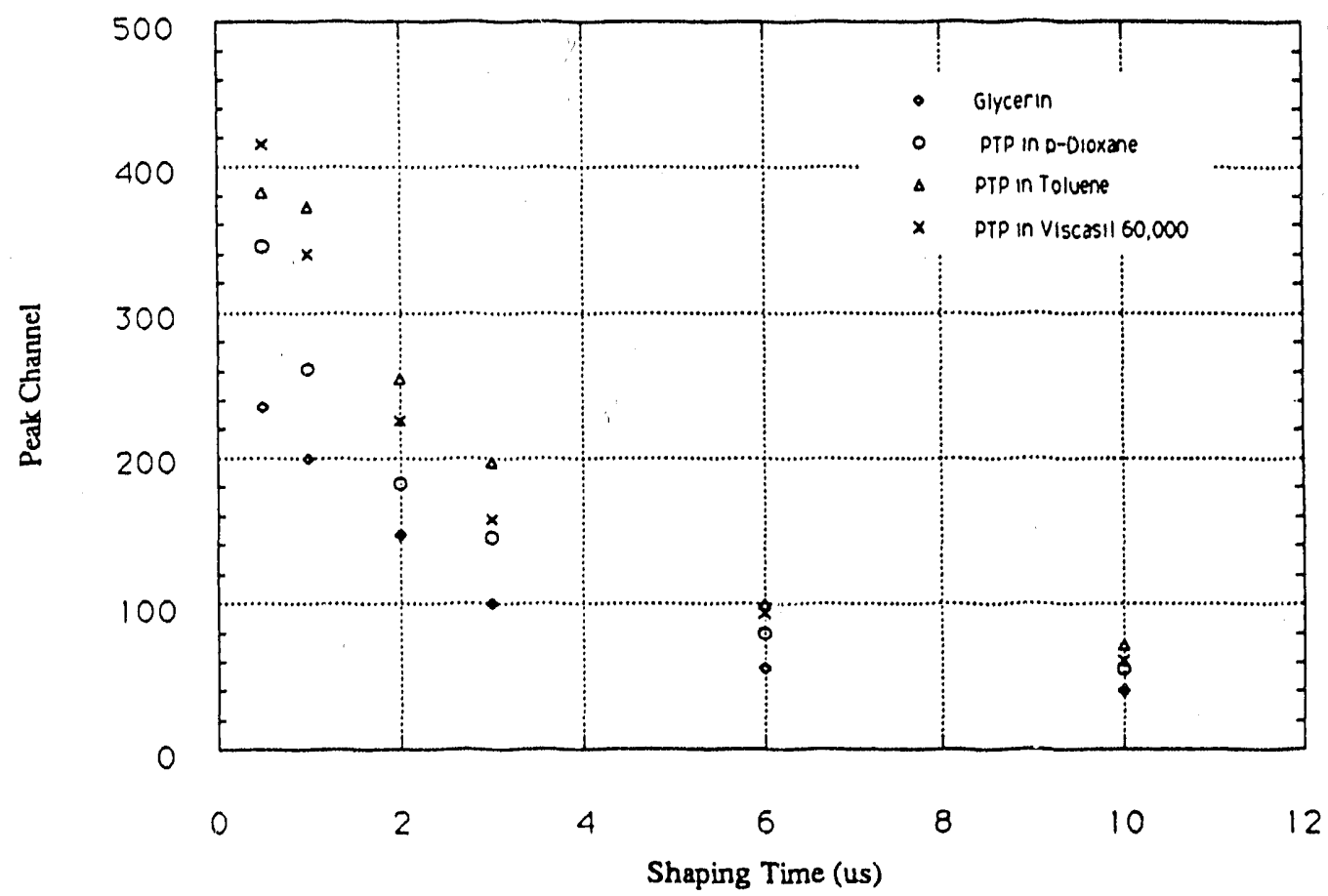

Figure 6. Peak channel normalized at each shaping time to a gain of $100 \mathrm{Vs}$. amplifier shaping time. Cs-137 irradiating a $2.54 \mathrm{~cm} \times 2.54 \mathrm{~cm}$ right circular cylinder of $\mathrm{BaF}_{2}$. The concentration of p-Terphenyl in the various solvents was $5 \mathrm{~m} / 1$. The wavelength shifter or glycerin occupied a space of $2.54 \mathrm{~cm}$ in diameter by $1 \mathrm{~cm}$ long between the scintillator and the XP2020 PMT. 


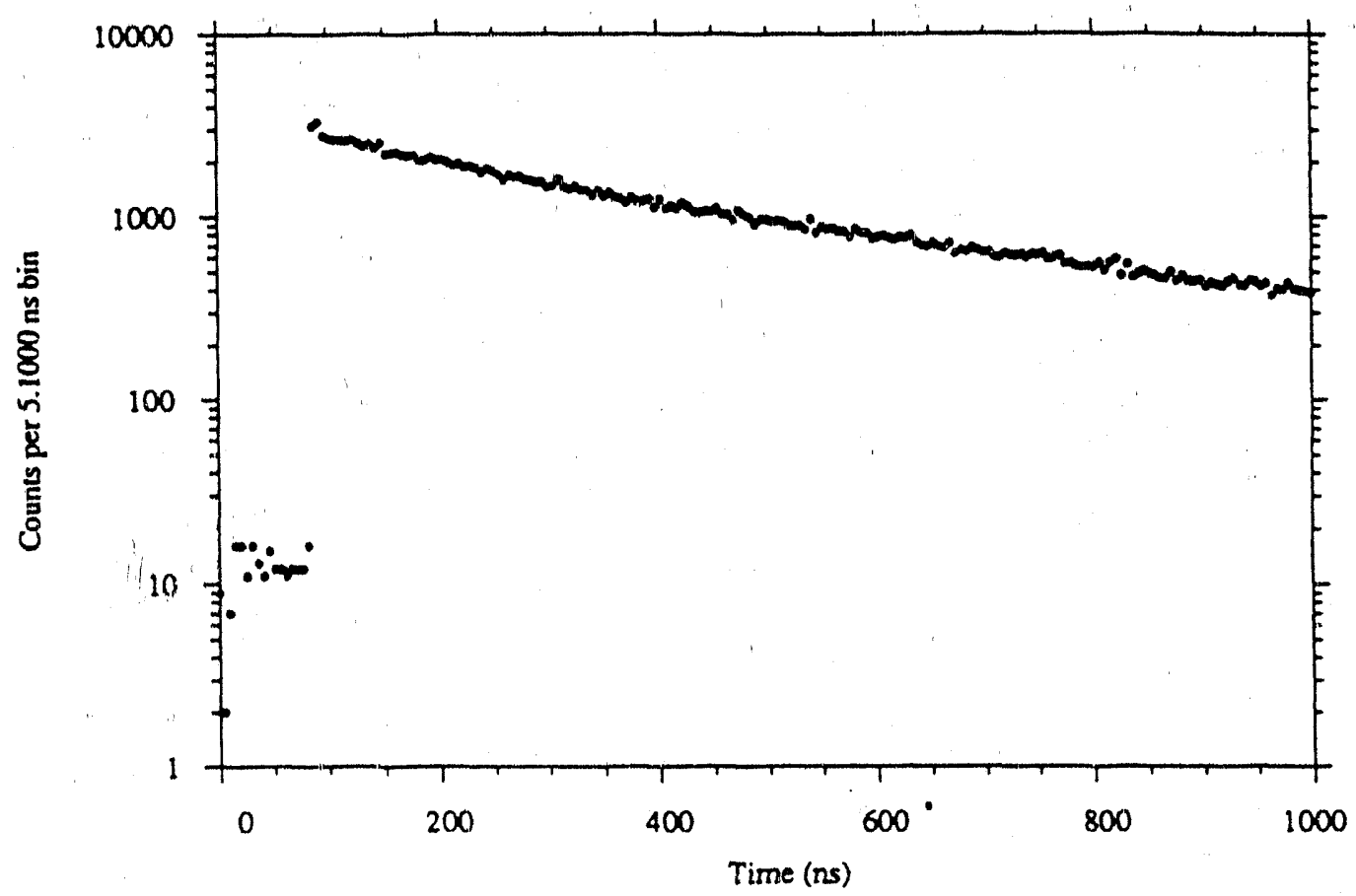

Figure 7. (a) The measured scintillation decay curve for $\mathrm{BaF}_{2}$ as measured with an XP2020 PMT. The XP2020 has a borosilicate end-window allowing only the slow 310 $\mathrm{nm}$ einission to be dotected. 


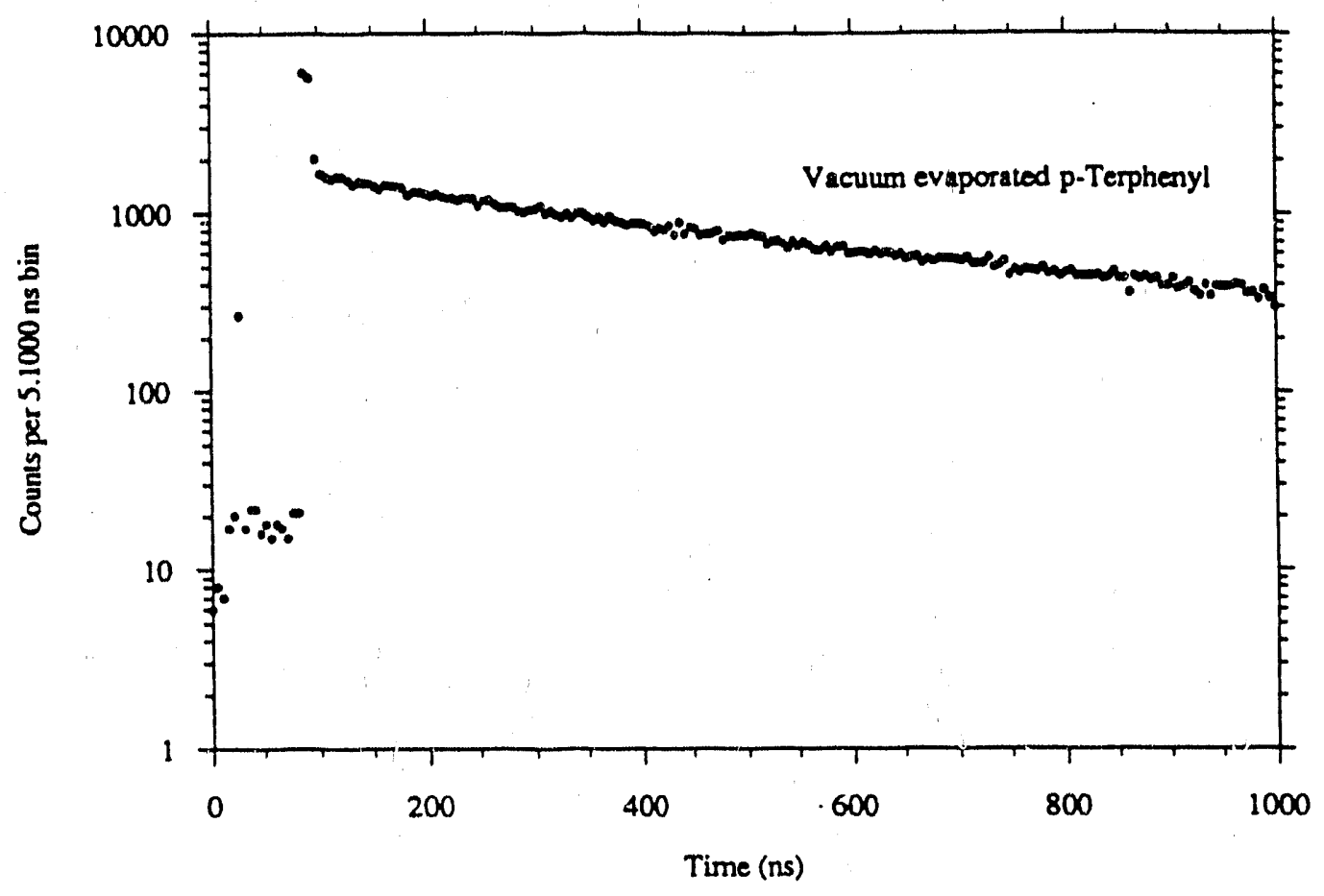

(b)

Figure $7(\mathrm{~b})$. A fast component is seen due to p-Terphenyl shifting the fast $220 \mathrm{~nm}$ emission to a wavelength that can be detected by the XP2020 PMT. The presence of the fast component is indicated by the spike at $90 \mathrm{~ns}$. 


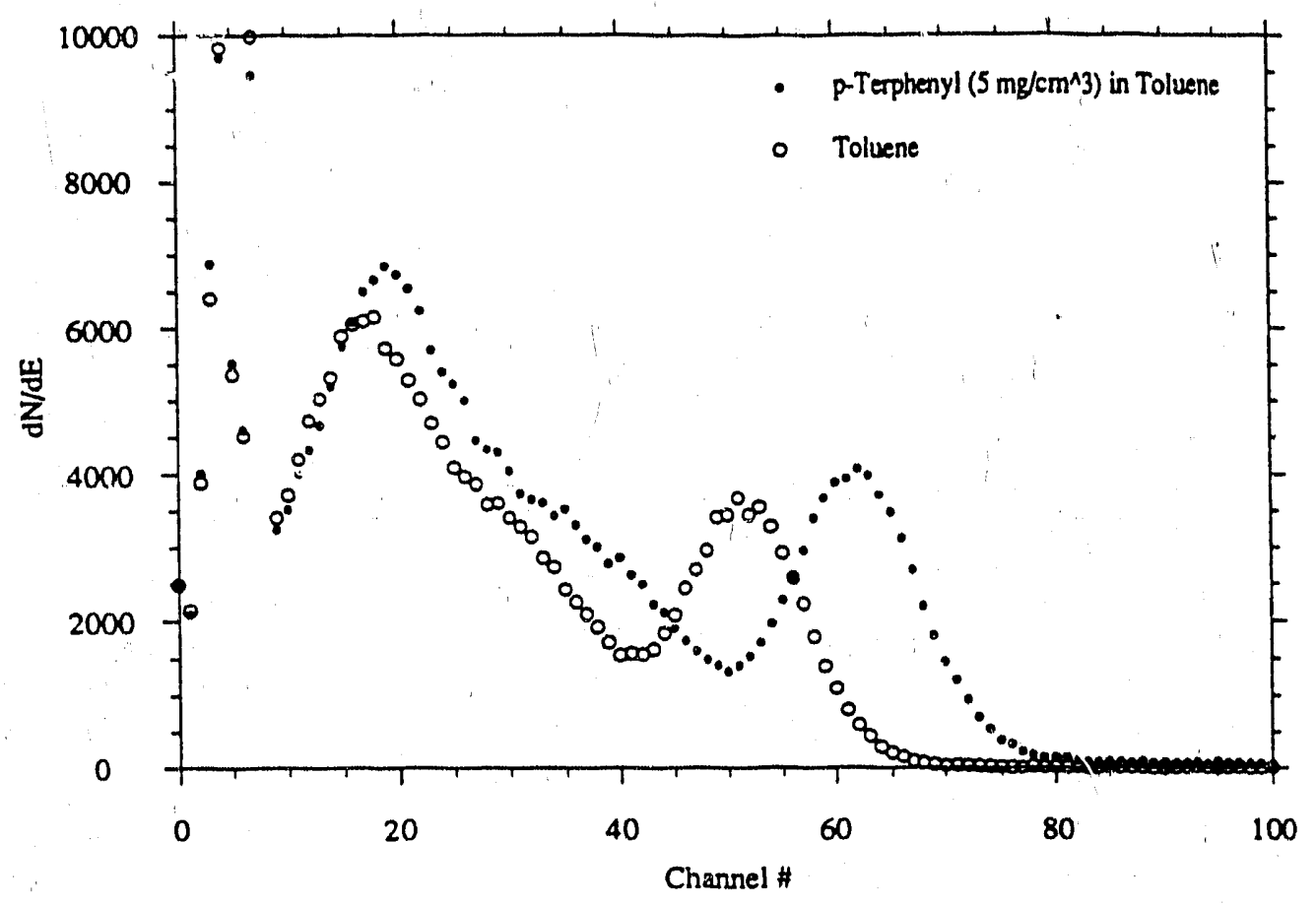

Figure 8. The pulse height spectrum for $66^{2} \mathrm{keV}$ as measured with a $2.54 \mathrm{~cm} \times 2.54 \mathrm{~cm}$ right circular cylinder of $\mathrm{BaF}_{2}$ and a XP2020 PMT. p-Terphenyl doped toluene shifts both the fast and the slow emission to $340 \mathrm{~nm}$ Coupling consists of $5 \mathrm{ml}$ of toluene or pTerphenyl-doped toluene.

As mentioned above, the introduction of the wavelength shifter will slow the decay time of the fast $\mathrm{BaF}_{2}$ emission. Although this slowing is significant, the wavelength shifted $\mathrm{BaF}_{2}$ is still suitable for count rate as high as $10^{7} \mathrm{cps}$. Table 1 shows the various organic dyes that we have investigited and their scintillation decay times. The role of the solvent can also be seen in the scintillation decay time for the various wavelength shifters.

Researchers have attempted to separate the fast and slow emissions of $\mathrm{BaF}_{2}$ using various methods, e.g... increasing crystal temperature, using special solar blind PMTs, and by introducing a dopant into the crystal. Our approach is to use wavelength shifting techniques to separate the fast and slow emissions of $\mathrm{BaF}_{2}$. The idea is to shift the fast component from $220 \mathrm{~nm}$ up to $\sim 450 \mathrm{~nm}$ and not to shift the $320 \mathrm{~nm}$ emission. Using a simple cutoff filter, the light that is incident on the PMT will only be due to the wavelength shifted fast component. Although other researchers have also recognized this approach, we believe we are the only group actively pursuing it. Table 2 shows the 
Table 1. Organic Dyes and Scindllation Decay Times

\begin{tabular}{|l|l|c|}
\hline Organic Dye & Solvent & Decay Time (ns) \\
\hline & & \\
\hline Coumarin 2 & Methanol & 4.04 \\
\hline Coumarin 2 & 1,4 Dioxane & 3.48 \\
\hline Coumarin 4 & 1,4 Dioxane & 2.44 \\
\hline Coumarin 120 & 1,4 Dioxane & 2.98 \\
\hline BM-Terpheny & Cyclohexane & 1.89 \\
\hline p-Terphenyl & & 2.69 \\
\hline p-Terphenyl & Silicone Oil & 2.94 \\
\hline p-Terphenyl & Toluene & 3.08 \\
\hline p-Terphenyl & 1,4 Dioxane & 2.64 \\
\hline Diphenylstilbene & & 3.40 \\
\hline
\end{tabular}

Table 2. Results of Wavelength Shifting

\begin{tabular}{|l|l|c|c|}
\hline Organic Dye & Solvent & Fast/Slow & *Luminosity \\
\hline & & & (in units of 108) \\
\hline Coumarin 2 & Methanol & 0.77 & 1.6 \\
\hline Coumarin 2 & 1,4 Dioxane & 0.94 & 2.6 \\
\hline Coumarin 4 & 1,4 Dioxane & 2.4 & 0.6 \\
\hline Coumarin 4 & Polyvinylalcohol & 1.16 & 2.7 \\
\hline Coumárin 120 & 1,4 Dioxane & 0.95 & 1.5 \\
\hline BM-Terphenyl & Cyclohexane & 0.35 & 1.4 \\
\hline BM-Terphenyl & 1,4 Dioxane & 0.39 & 1.7 \\
\hline BM-Terphenyl & Toluene & 0.15 & 0.7 \\
\hline p-Terphenyl & & 1.1 & 1.4 \\
\hline p-Terphenyl & Toluene & 0.32 & 0.6 \\
\hline p-Terphenyl & 1,4 Dioxane & 0.48 & 1.0 \\
\hline Diphenylstilbene & & 0.33 & 1.3 \\
\hline
\end{tabular}

* Luminosity was determined by determining the counts under the emission peak when the sample is excited with $220 \mathrm{~nm}$ light. The peak area is then corrected for the different operating voltages that were used. Monochrometer step size was $1 \mathrm{~nm}$ and the count rate w/as determined at each step by integrating for 1 second. 
results of the wavelength shifters studied during the past year. The fast to slow ratio stated was obtained by exciting with an equal photon intensity of either $220 \mathrm{~nm}$ or 310 $\mathrm{nm}$. This was done by bandpass filtering the emission of a deuterium lartip

From the results found in table 2 and noting the fast light comprises only $20 \%$ of the total light output, we can conclude $10 \mathrm{~g} / \mathrm{l}$ of Cournarin 4 in 1,4 Dioxane, used as a wavelength shifter for $\mathrm{BaF2}$, will result in a fast to slow ratio of 0.4 for the $\mathrm{BaF} /$ wavelength shifter system. However, cther researchers have been able to obtain a ratio closer to 1.0 by using La-doped $\mathrm{BaF} 2$. While we are pleased with our enhanced fast/slow ratio, we believe continued research may lead to our design goal of a fast/slow ratio of 10 .

\subsection{Electronically-Collimated Cameras}

\subsection{Theoretical design}

Gamma ray cameras with good energy and position resolution are needed to locate weak and diffuse radioactive sources and characterize the gamma-ray spectra. The concept of Compton scatter imaging and its possible applications weje first suggested by Everet ${ }^{[2]}$. An electronically collimated gamma camera $(E C C)$ was one of the first working, cameras based on this principle. The ECC was introduced in 1974, and subsequent work, primarily by Singh, led ultimately to a working prototype for medical imaging[3].

As an extension of the electronic collimation concept, Kamea and Hanada ${ }^{[4,5]}$ introduced a new method called the multiple Compton method. A multiple Compton camera consists of a series of large area thin silicon (or germanium) layers. Each layer is an independent, two dimensional, position sensitive detector. The layers are designed to be sufficiently thin so the probability of multiple interactions in any layer is small. If the stack is made sufficiently large, there is a reasonable probability that an incoming gamma ray, after a series of Compton scatterings, will be photoabsorbed in one of the layers. Alternately, the stack can be surrounded with a high-efficiency annular detector (a highdensity scintillator) to intercept those photons that escape the stack without photoabsorption. Thus, each individual interaction position and energy deposition from either a Compton scattering or photoelectric absorption in the stack is recorded. The energy and polar direction of the incoming photon can usually be obtained from the interaction positions and energy depositions along its history, whether in the stack or in the surrounding annular detector. 
While the multiple Compton camera is substantially more complex than the single $E C C$, it offers the potential of high efficiency measurements of the polar direction, polarization, and energy of the incident gamma rays. In our first year's work, we explored the multiple Compton camera concept using Monte Carlo simulations of both $\mathrm{Si}$ and Ge layers. During this second year, we hove turned our attention to the single scatter camera. In particular, we have explored new algorithms for image processing, acquired the first detector of a single scatter camera, examined a means to exploit the polarization dependence of the multiple Compton camera for image processing, and began acquisition of the second detector element necessary to complete the imaging system.

\subsubsection{Single Compion Camera Work}

A diagram of a single scatter Compton camera geometry is shown in Figure 9. An incident gamma ray undergoes Compton scatter in the first detector, and then interacts in the second. The energy of the recoil electron is measured in the first detector and the direction of the scattered gamma ray is determined from the positions of the interactions in the two detectors. With the energy of the incident gamma ray determined from the total energy deposition, the direction can be determined (with azimuthal ambiguity) from this measurement. The angular resolution is a function of the position and energy resolution of both detectors.

\section{Ge Detector Array}

During this past year, we have acquired an $4 \times 4$ array of 16 germanium crysta's to be used as the first detector in our Compton imaging camera. Each of the crystals has a $5 \times 5 \mathrm{~mm}$ square surface and a depth of $6 \mathrm{~mm}$. The array is housed in an evacuated cryostat which is cooled with liquid nitrogen to $77 \mathrm{~K}$. The vacuum is maintained with an ion pump. Figure 10 is a photograph of the array housing; the location of the crystals is etched on the front of this housing.

Figure 11 is a photograph of the opposite side of the array housing. The inner circular plate is the cryostat. In addition to the Ge crystals themselves, field effect transistors, which form the first stage of the signal processing electronics, reside in the cryostat to help improve energy resolution. Positioned around the cryostat are the 16 miniature preamplifier boards, one for each crystal. Power for the preamplifiers and the output signals are fed through ribbon cables to two NIM, 8 channel fast amplifiers. Figure 12 is a schematic of the signal processing electronics for the Ge detector array.

The NIM amplifiers provide shaped, amplified signals to a rack mounted digital logic module. This module digitizes each pulse height signal in six bit flash analog to 


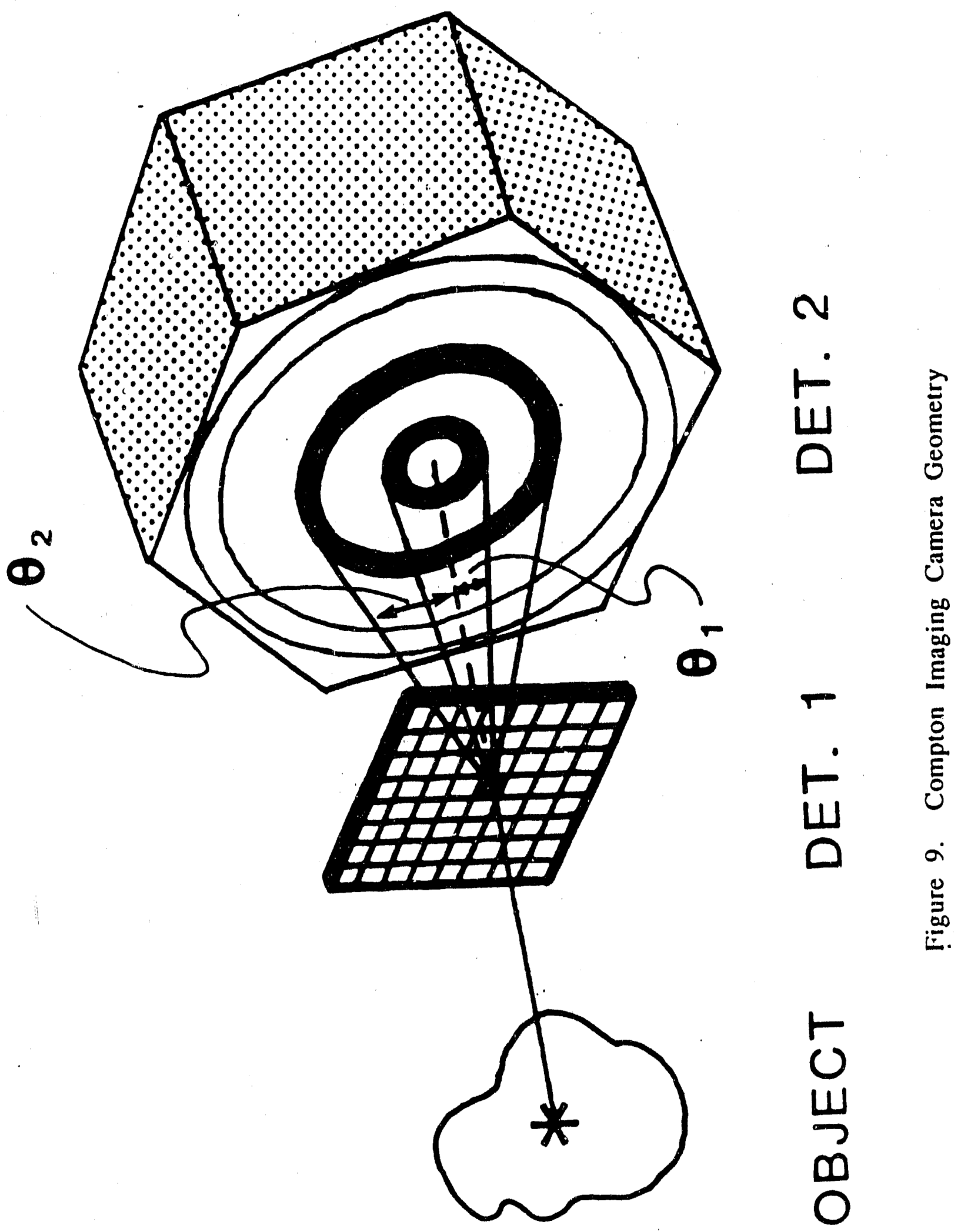




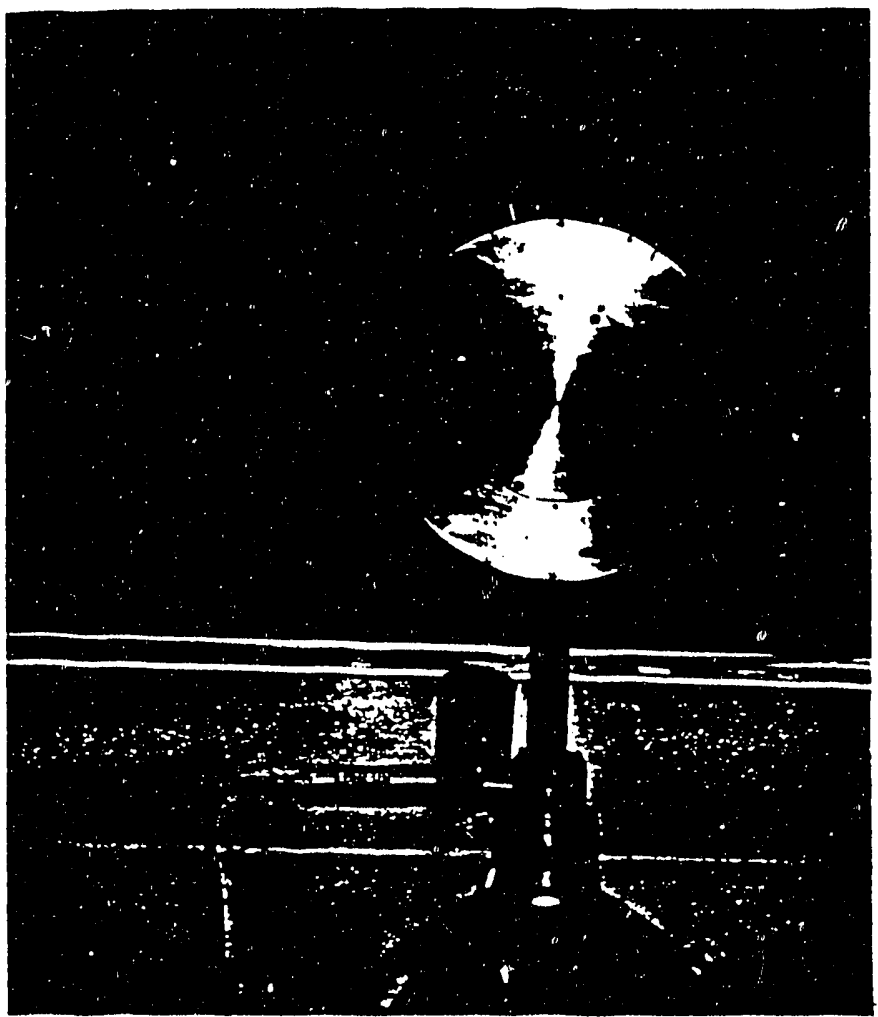

Figure 10. Photograph of Ge Array Detector and Support Equipment

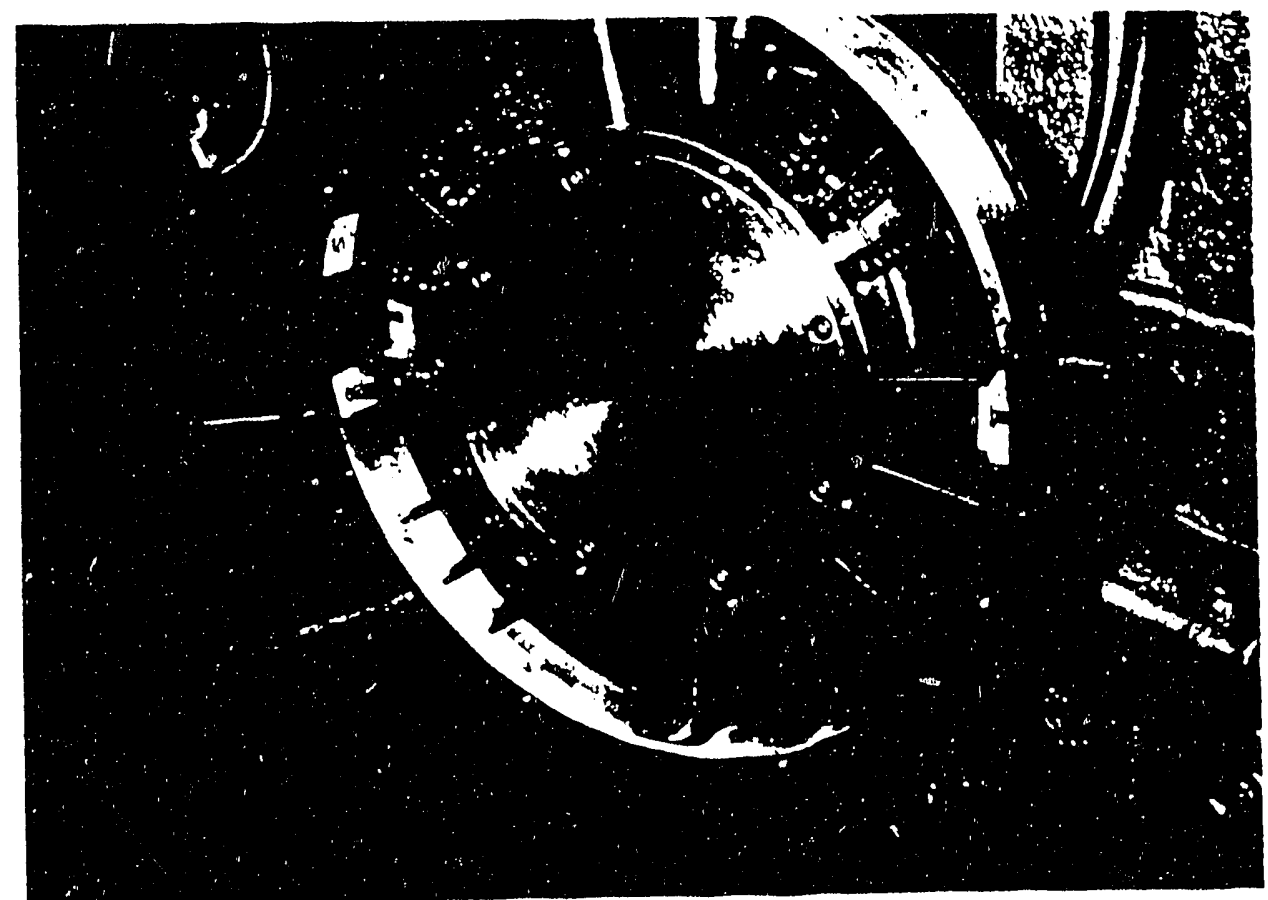

Figure 11. Photograph of Ge Array Housing, Reverse View 


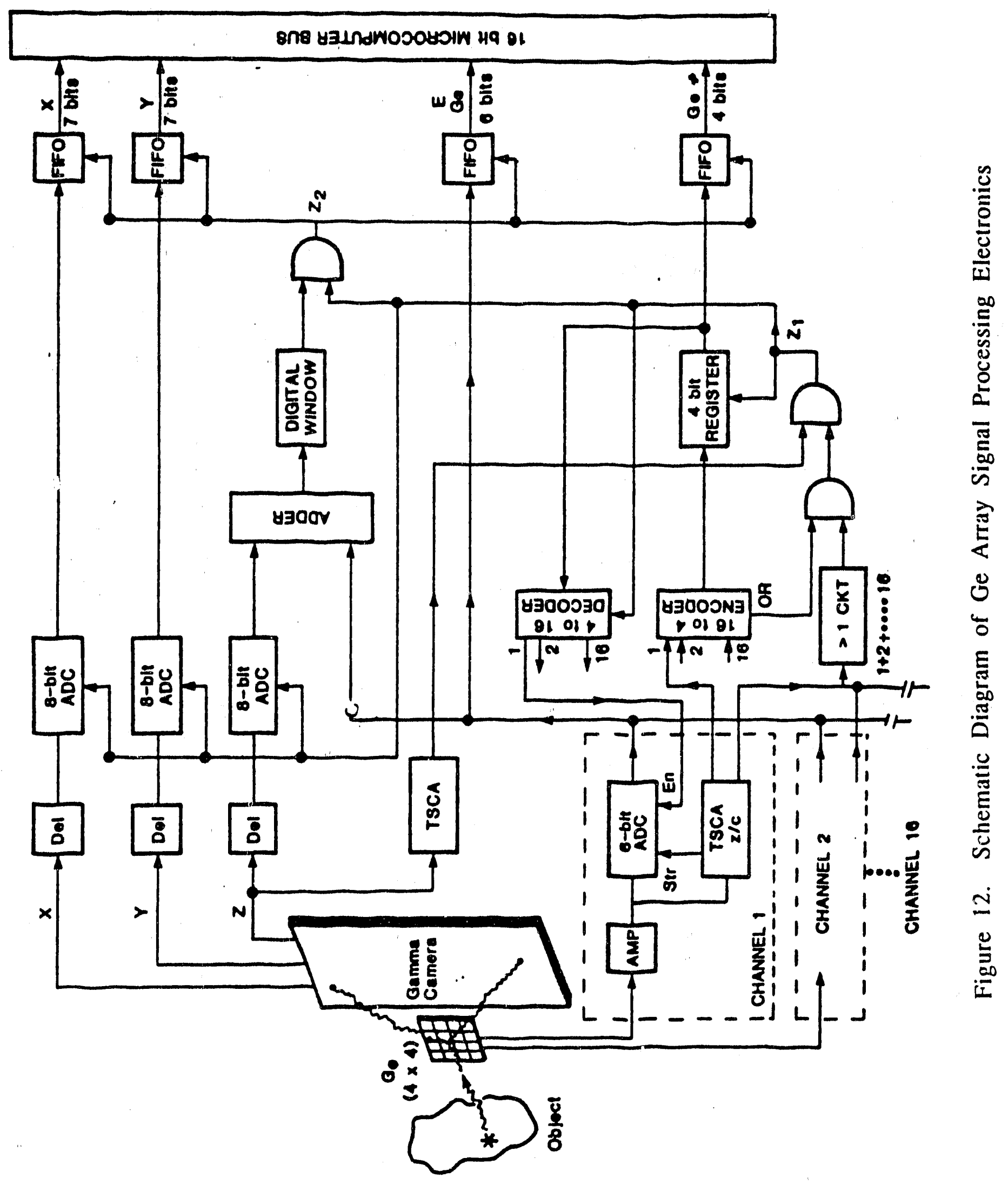


digital converters and encodes the crystal where the signal originated as a four bit word. The module also provides a time coincidence window between the array and the second detector when the timing signal is provided from the second detector.

\section{Effect of Detector Energy Resolution on Image Resolution.}

The spatial resolution of two dimensional images measured with a Compton camera is proportional to the distance from the source to the first detector. As a result, spatial resolution in a Compton camera can be represented more meaningfully by its angular resolution. The total angular resolution, $\Delta \theta$ can be expressed as a function of the system energy resolution, $\Delta \theta_{\mathrm{E}}$, and position resolution of the first, $\Delta \theta_{\mathrm{P} 1}$, and second, $\Delta \theta_{\mathrm{p} 2}$, detectors.

$$
\tan ^{2}(\Delta \theta)=\tan ^{2}\left(\Delta \theta_{E}\right)+\tan ^{2}\left(\Delta \theta_{P 1}\right)+\tan ^{2}\left(\Delta \theta_{P 2}\right)
$$

The quadrature sum of the energy resolution in the first and second detector yield the energy resolution of the system.

$$
\left(\Delta \theta_{E}\right)^{2}=\left(\Delta \theta_{E 1}\right)^{2}+\left(\Delta \theta_{E 2}\right)^{2}
$$

However, if the incident gamma ray energy is known, the energy of the gamma ray in the second detector can be determined by the energy measured in the first, and $\Delta \theta_{\mathrm{E}}$ depends only on $\Delta \theta_{\mathrm{El}}$.

We have studied the dependence of angular resolution on the energy resolution of the first detector. This dependence can be expressed as:

$$
\left(\Delta \theta_{E_{1}}\right)^{2}=\left(\mathrm{d} \theta / \mathrm{dE}_{1}\right)^{2}\left(\Delta \mathrm{E}_{1}\right)^{2}
$$

or,

$$
\Delta \theta_{\mathrm{E} 1}=\Delta \mathrm{E}_{1} / \mathrm{dE_{1 }} / \mathrm{d} \theta
$$

where,

$$
\begin{aligned}
& d E_{1} / d \theta=\sin (\theta)\left(E_{\gamma} E_{1}\right)^{2} / m_{0} c^{2} \\
& \theta=\text { scattering angle } \\
& E_{1}=\text { energy deposited in first detector = recoil electron energy } \\
& E_{\gamma}=\text { incident gamma ray energy }
\end{aligned}
$$

The minimum angular resolution results where $l d E_{1} / d \theta \mid$ reaches a maximum. Figure 13 shows how $\mathrm{dE}_{1} / \mathrm{d} \theta$ varies as a function of $\theta$, for a range of incident gamma ray energies. 


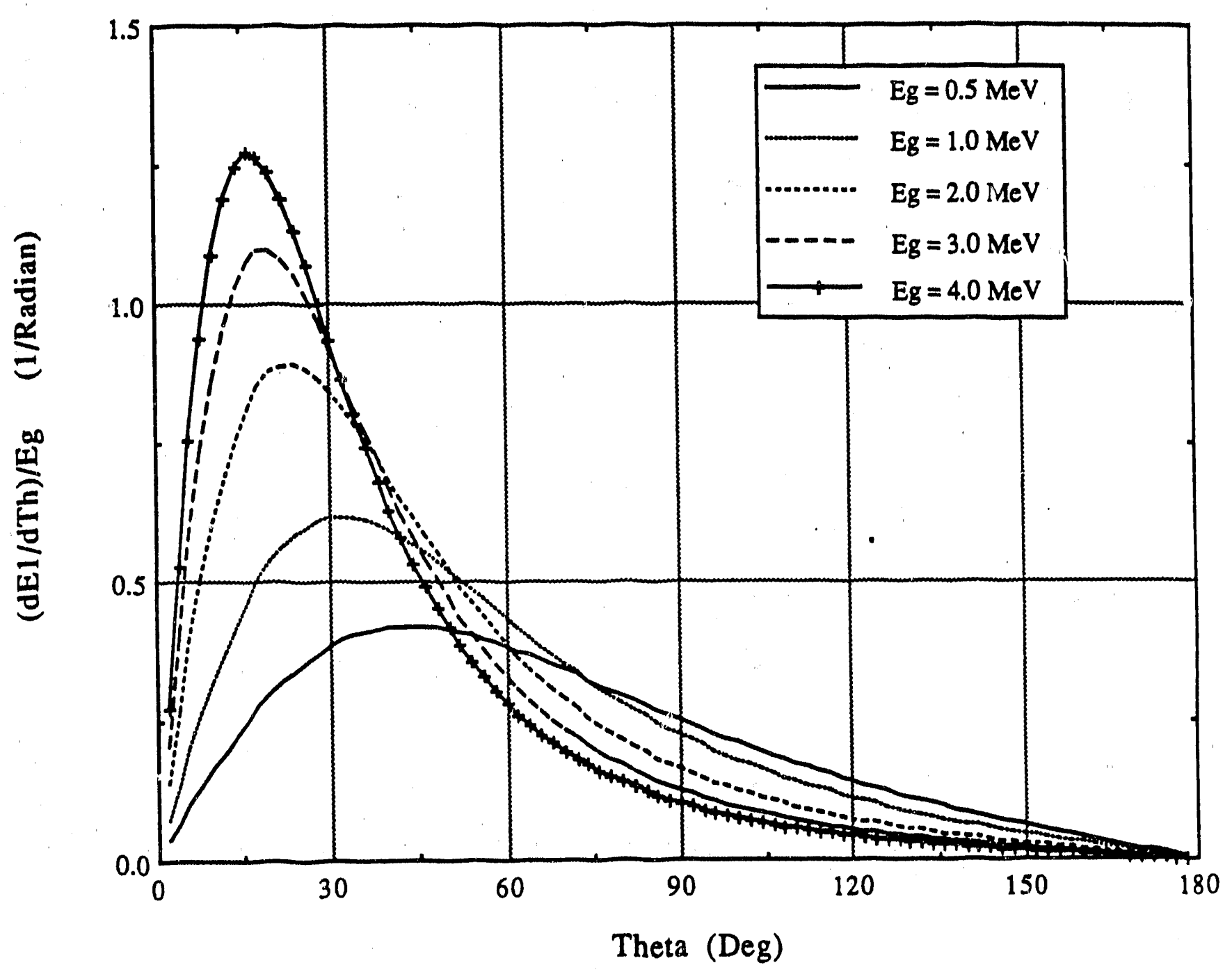

Figure 13. Fractional Change of E1 with Theta as a Function of Theta for a Range of Incident Gamma-Ray Energies 
This figure indicates that to optimize the resolution of a Compton camera, highly forward scattering angles and all backward scattering angles should be avoided.

From these results, we have concluded that the best angular resolution for a Compton camera will be realized if the second detector has a ring geometry. This geometry allows for selection of a limited range of scattering angles where $\left|d E_{1} / d \theta\right|$ is large, but the full ring will subiend a large solid angle.

\section{Ring Array of NaI Crystals.}

We currently have a ring of $78 \mathrm{NaI}(\Pi)$ crystals available for use as the second detector in our Compton camera. This system (called SPRINT) was designed as a single photon imaging medical tomography machine. The ring diameter is $734 \mathrm{~mm}$, and each crystal is a $19 \mathrm{~mm}$ diameter by $51 \mathrm{~mm}$ long cylinder. SPKINT is approximately 10 years old, and the condition of the $\mathrm{NaI}$ crystals and associated electronics has not been evaluated yet. Another system (called ASP) is also available for use as a second detector. The renewal proposal discusses the relative merits of each of these two available systems.

\subsubsection{Image Formation from the Interaction Data}

As mentioned above, a single Compton scattering followed by a photoabsorption only yields the cone on which the gamma ray was incident. As a result, one must deconvolve the image from the measured data. Herbert $(6)$ has proposed one such algorithm using a maximum likelihood principle. However, the algorithm is too computationally intensive to produce images in near real-time. We have developed two algorithms that promise improved efficiency in translating the interaction data into images. We describe these methods briefly below.

In the first algorithm, we discretize all possible incoming directions $\vec{k}_{0}$ and observe the $\vec{k}_{1}$ scattered photons. We then increment counts for all incident photon directions which satisfy the relationship:

$$
\vec{k}_{0} \bullet \vec{k}_{1}=\frac{\cos \Theta}{\left|\overrightarrow{k_{0}}\right|\left|\overrightarrow{k_{1}}\right|}
$$

This technique is similar to the "event circle" method used in astrophysics(7). The disadvantage of the method is a relatively poor signal to noise ratio since many false incident vectors are recorded for each true incident vector Nevertheless, the technique is simple to implement and computationally fast.

In the second method, we discretize all possible Compton scattering cones. We then examine all possible intersection of these cones. Since a single scattering gives rise 
to one cone, two gamma rays originating from the same location will have cones which intersect along the incident direction. Thus, by comparing these onnes, one can isolate the incident source directions. The method has excellent signal to noise ratio properties, but is computationally more intensive since it involves solution of a quadratic equation. Fortunately, most of the calculations can be performed a priori and only table lookups are required during image acquisition.

\subsubsection{Computer Simulation Results}

The sample camera design has been tested using the Integrated Tiger Series (ITS) puckage of Monte Carlo codes [8] which we described in our previous progress report. In the simulation, several point sources were placed away from the camera and simulated data were acquired. Figures 14 and 15 show the results of the simulation. Both methods yield peaks which are located correctly at the source locations. The relative intensities of the individual peaks are not meaningful since we have not corrected for the detection efficiency as a function of source position and energy. As expected, the intersection method yielded better defined point sources and introduced negligible background. Because of this small background, well defined images may be able to be produced with many fewer incident photons. Current efforts are evaluating the effectiveness of this algorithm for distributed sources.

\subsection{Reducing Azimuthal Ambiguity for Double Compton Scattering through Polarization Information}

As roted above, reconstruction of the initial direction of the incident gamma ray can be performed only to within a $2 \pi$ azimuthal angle ambiguity since the Compton scattering formula contains no information on the azimuthal angle. This is analogous to medical PET in which each coincident $.511 \mathrm{MeV}$ annihilation pair yields only the line on which the emitting nucleus exists. In the case of PET, this ambiguity can be reduced through TOF information. This additional information, although containing poor spatial resolution, permits an improvement in the PET image reconstruction. For our case, similar information exists through a more careful consideration of gamma ray polarization effects.

The quantum mechanics associated with the interactions of polarized and unpolarized photons with materials is extremely complex and the derivations will not be reproduced here. For double Compton scattering, the three momentum vectors form two 

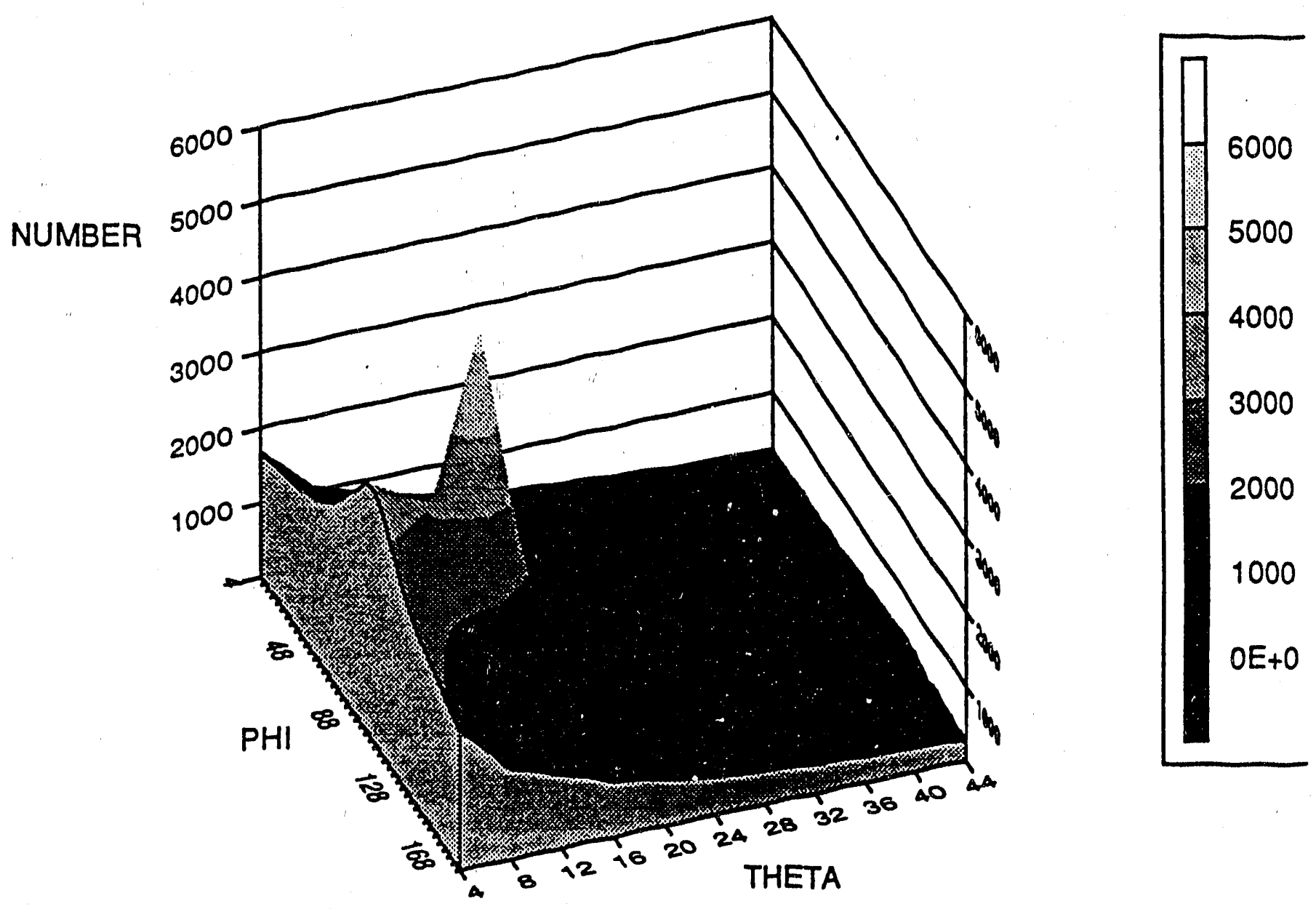

Figure 14. Incoming Gamma Ray Directions with Event Cone Method 


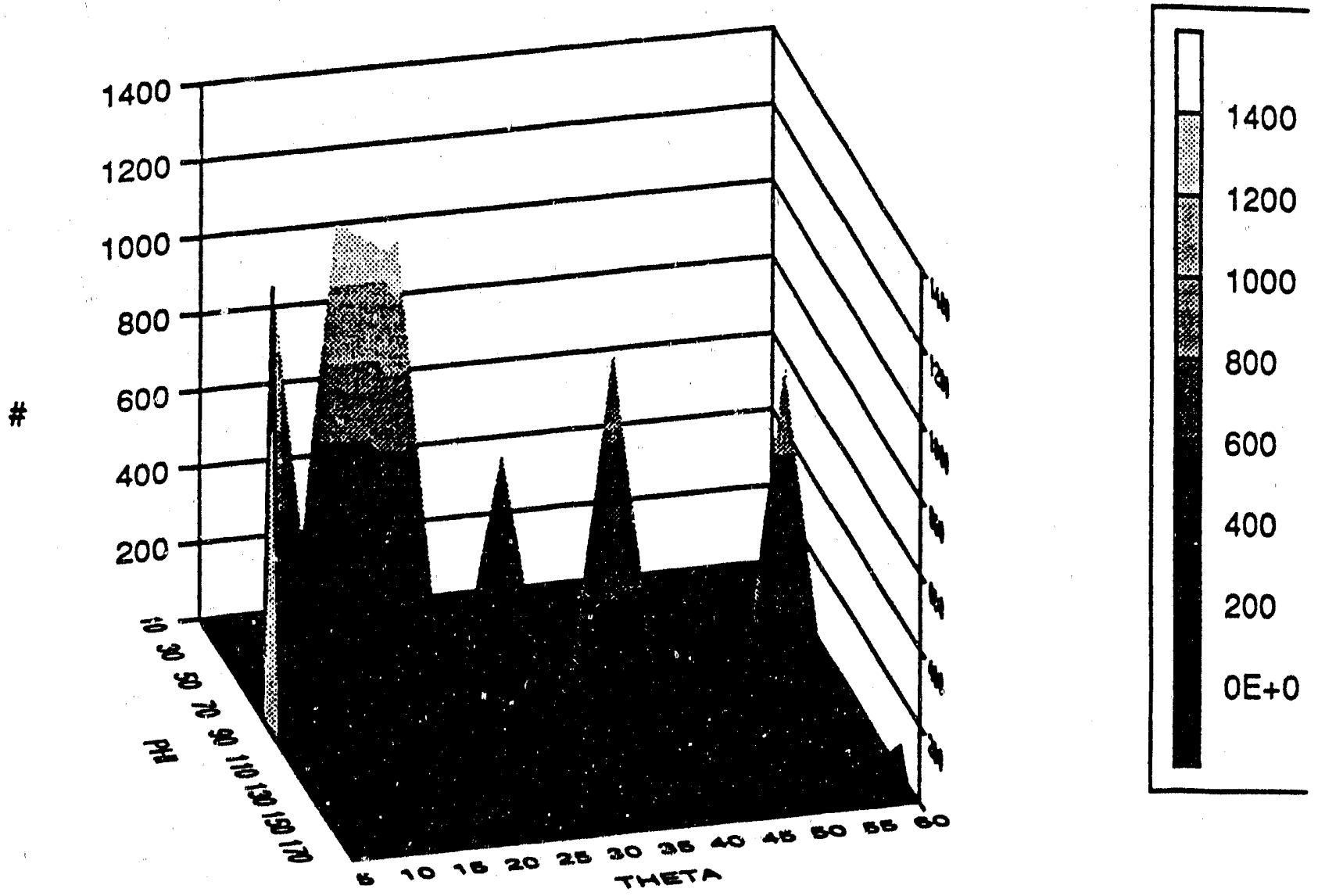

Figure 15. Incoming Gamma Ray Directions from Intersection of the Cones 
planes with angle $\phi$ between them. Assuming the incident gamma rays are unpolarized, a careful derivation of the double Compton scattering cross section formula shows an azimuthal angle $\phi$ dependence of the cross section:

$\frac{d \sigma}{d \Omega}=\left(\frac{1}{4}\right) r_{0}^{4} \quad\left(k_{2} / k_{0}\right)^{2} \quad\left(\gamma_{01} \gamma_{12}-\gamma_{01} \sin ^{2} \theta_{2}-\lambda_{12} \sin ^{2} \theta_{1}+2 \sin ^{2} \theta_{1} \sin ^{2} \theta_{2} \operatorname{Cos}^{2} \Phi\right)$

where the variables are defined in Table 3. Note the explicit dependence of the cross section on the azimuthal angle $\phi$. Similar formulas can be written for higher order Compton scattering for the multiple scatter camera.

\begin{tabular}{|c|c|}
\hline Variable & Meaning \\
\hline$r_{0}$ & classical radius of electron \\
\hline$\theta_{j}$ & $\mathrm{j}^{\text {th }}$ Compton scattering polar angle \\
\hline$k_{0}$ & incident gamma ray energy \\
\hline $\mathrm{k}_{\mathrm{j}}$ & energy of gamma ray after $j^{\text {th }}$ scattering \\
\hline$\gamma_{i j}$ & $\mathrm{k}_{\mathrm{i}} / \mathrm{k}_{\mathrm{j}}+\mathrm{k}_{\mathrm{j}} / \mathrm{k}_{\mathrm{i}}$ \\
\hline$\phi_{\mathrm{j}}$ & $\begin{array}{l}\text { angle between planes containing vectors } \\
\left(\mathrm{k}_{\mathrm{i}-1}, \mathrm{k}_{\mathrm{j}}\right) \text { and }\left(\mathrm{k}_{\mathrm{i}}, \mathrm{k}_{\mathrm{i}+1}\right)\end{array}$ \\
\hline
\end{tabular}

As can be seen from equation (1), the impact of polarization is greatest for Compton scattering angles closest to 90 degrees and at lower energies. The additional information offered by polarization effects will be strongly dependent on the actual Compton scattering angles. Although we have not considered it yet, this additional polarization information may also help to unfold the correct sequence of scatterings.

During the past year, we incorporated polarization effects into the ITS Monte Carlo code package. Using this as our data source, we $\operatorname{ran} 500,000$ unpolarized photons through a simulated Compton camera. The double scattering data were then used to test a new algorithm based upon equation (1). In this method, an arbitrary reference direction is chosen, and all scatterings with similar $k_{1}$ vectors are grouped. As shown in Figures 16 and 17 , the distribution of $k_{2}$ vectors then yields the azimuthal direction of the incident gamma rays relative to the arbitrary reference direction. Table 4 shows the results of changing the arbitrary reference direction. Note that the distribution, whether measured using $\cos \phi$ or $\cos ^{2} \phi$, yields quite accurate results. 


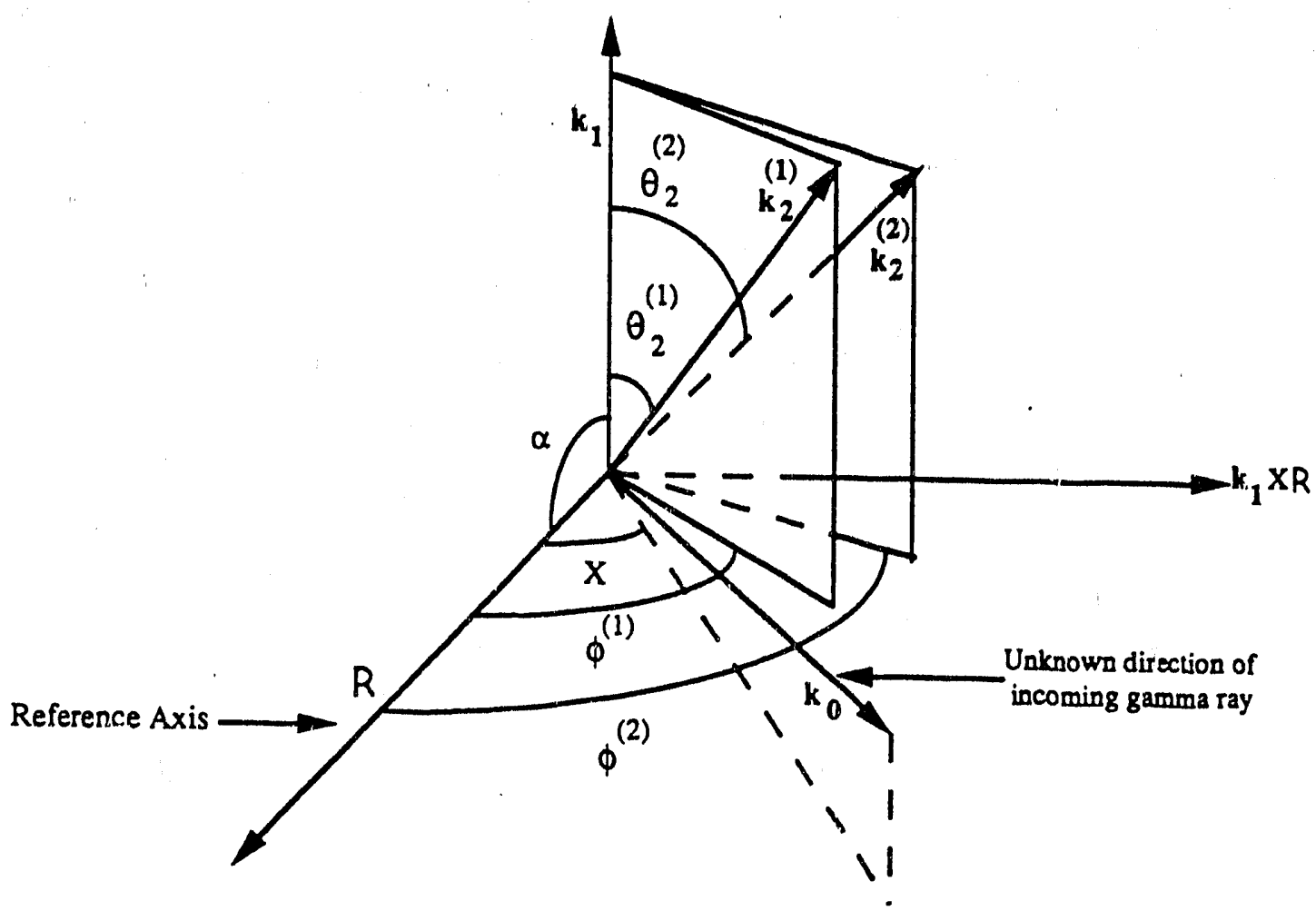

Figure 16. The Direction Vectors Involved in Double Compton Scattering 


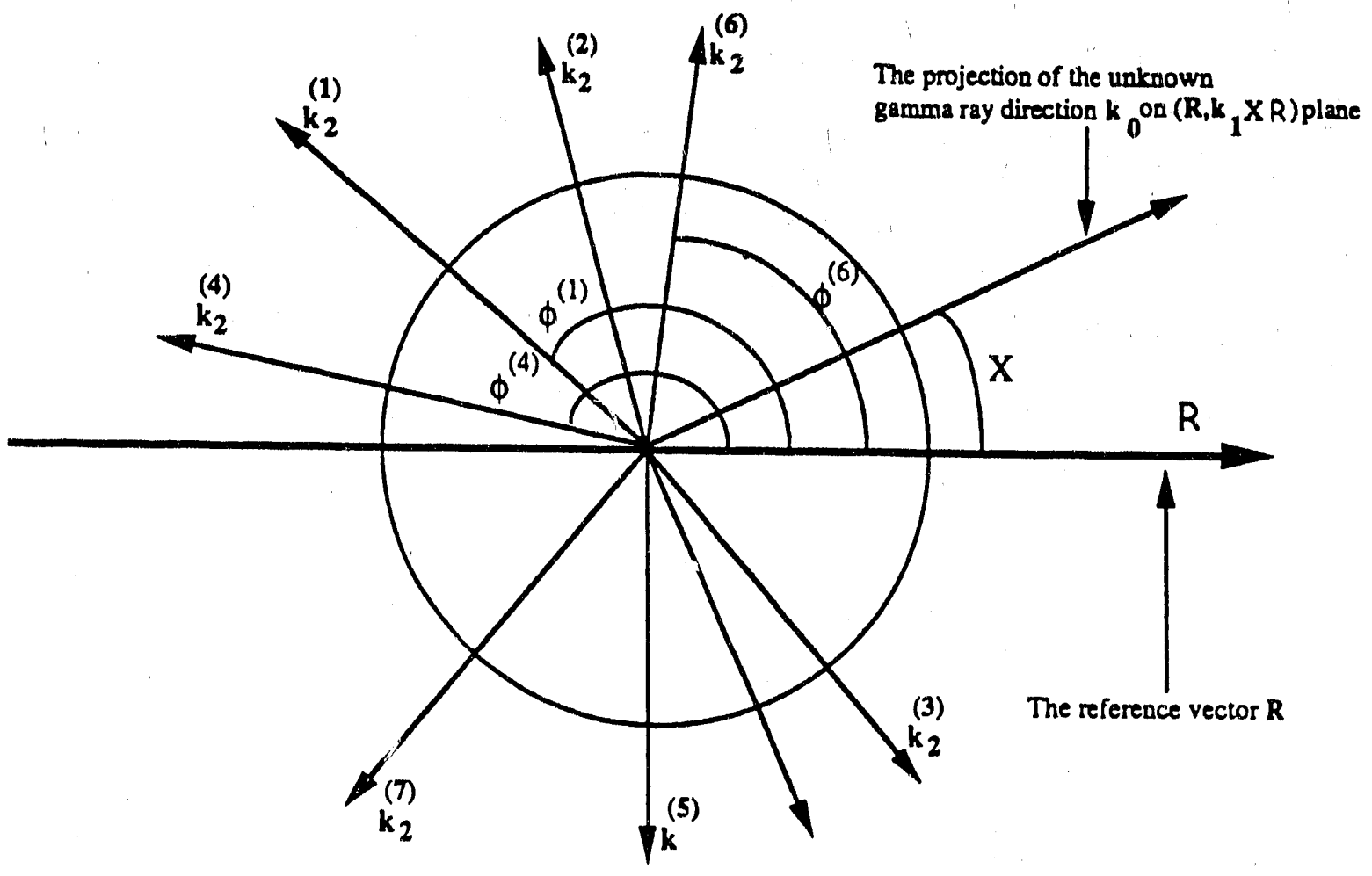

Figure 17. The top View of the Direction vectors in Fig. 16. 
Table 4. Results from 2D-CYLTRAN for 500,000 Photon History

\begin{tabular}{|l|c|c|}
\hline $\begin{array}{c}\text { Reference Axis } \\
\text { Orientation }\end{array}$ & $X(0)$ from $\cos \phi$ & $X\left({ }^{0}\right)$ from $\cos ^{2} \phi$ \\
\hline $\begin{array}{l}\text { Parallel to he incoming } \\
\text { gamma ray direction }\end{array}$ & 6.705 & 0.0 \\
\hline $\begin{array}{l}30 \text { degrees respect to the } \\
\text { incoming gamma ray direction }\end{array}$ & 32.87 & 31.99 \\
\hline $\begin{array}{l}45 \text { degrees respect to the } \\
\text { incoming gamma ray direction }\end{array}$ & 45.0 & 45.25 \\
\hline $\begin{array}{l}75 \text { degrees respect to the } \\
\text { incoming gamma ray direction }\end{array}$ & 86.31 & 72.09 \\
\hline $\begin{array}{l}\text { Perpendicular to the incoming } \\
\text { gamma ray direction }\end{array}$ & 87.15 & 90.0 \\
\hline
\end{tabular}




\subsection{Summary}

In the development of th: mechanically-collimated camera, we have designed, built, and tested the first prototype radiation imaging camera. We have demonstrated its capabilities using laboratory sources, as well as under practical environmental conditions in our reactor and in hot caves. We have also designed the second generation device, and are proceeding with its fabrication.

While the MCC design has been proven, we believe the most exciting developments remain ahead for the electronically collimated design. We have acquired the first element of a single Compton camera, and are awaiting the delivery of the second detector elements. While the transformation of this hardware into a working camera will involve a major effor, our theoretical studies indicate that the potential for this type of camera will exceed that of the MCC for many practical applications involving common radiation fields.

\subsection{References}

1. D. Dolin, T. DeVol, and D. K. Wehe, "Multichannel Spectrum Analysis for a GammaRay Imaging Camera," Trans. Amer. Nucl. Soc., 63, 132(1991).

2. D. B. Everet, et al., Proc. IEEE, 124, 995(1977).

3. M. Singh and D. Doria, "An Electronically Collimated Gamma Camera for Single Gamma Emission Computed Tomography," Medical Physics, 10, 421(1983).

4. T. Kamea, et al., "Energy, direction and polarization of gamma rays," Nucl. Instr. and Melh., A260, 254(1987).

5. T. Kamea, H. Hanada, "Prototype Design of Multiple Compton Gamma Ray Camera," IEEE Transactions on Nuclear Science, 35, No. 1, 352(1988).

6. T. Herbert, R. Leahy, and M. Singh, "Three-dimensional maximum-likelihood reconstruction for an electronically collimated single-photon-emission imaging system," J. Opt. Soc. Amer., 7, No. 7, July, 1990.

7. V. Schonfelder, U. Graser, and R. Diehl, "Properties and Performance of the MPI Balloon Borne Compton Telescope," Astron. Astrophys., 110, 138(1982).

8. A. Halbleib, T. A. Melhorn, "The Integrated TIGER Series of Coupled Electron/Photon Monte Carlo Transport Codes," Sandia National Laboratory (1984). 


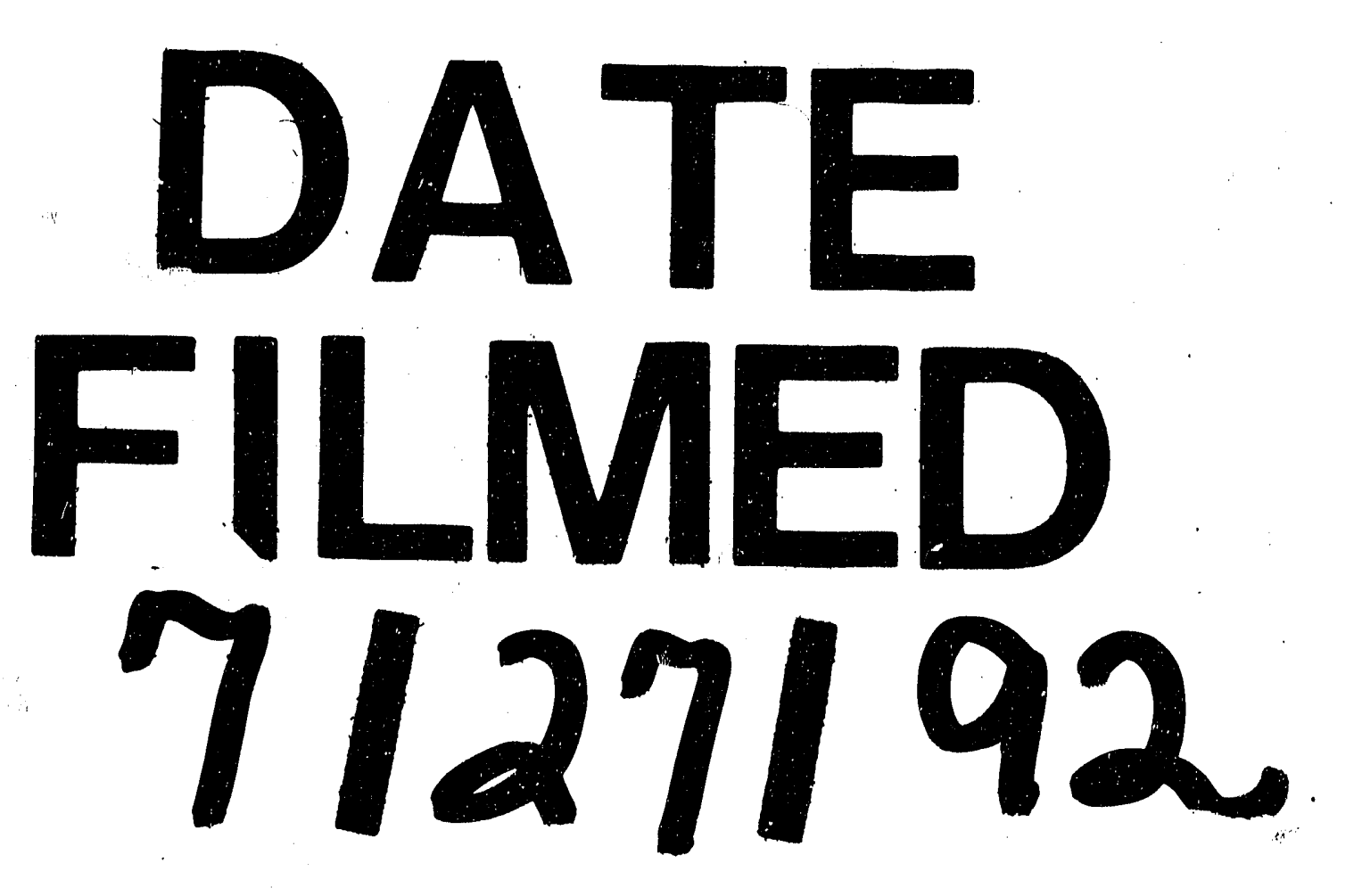


